\title{
Weighted Morrey Spaces Related to Schrödinger Operators with Nonnegative Potentials and Fractional Integrals
}

\author{
Hua Wang $\mathbb{D}^{1,2}$ \\ ${ }^{1}$ School of Mathematics and Systems Science, Xinjiang University, Urumqi 830046, China \\ ${ }^{2}$ Department of Mathematics and Statistics, Memorial University, Nl A1C 5S7, St. John's, Canada
}

Correspondence should be addressed to Hua Wang; wanghua@pku.edu.cn

Received 22 June 2019; Accepted 21 November 2019; Published 16 March 2020

Academic Editor: Yongsheng S. Han

Copyright (c) 2020 Hua Wang. This is an open access article distributed under the Creative Commons Attribution License, which permits unrestricted use, distribution, and reproduction in any medium, provided the original work is properly cited.

Let $\mathscr{L}=-\Delta+V$ be a Schrödinger operator on $\mathbb{R}^{d}, d \geq 3$, where $\Delta$ is the Laplacian operator on $\mathbb{R}^{d}$, and the nonnegative potential $V$ belongs to the reverse Hölder class $\mathrm{RH}_{s}$ with $s \geq d / 2$. For given $0<\alpha<d$, the fractional integrals associated with the Schrödinger operator $\mathscr{L}$ is defined by $\mathscr{I}_{\alpha}=\mathscr{L}^{-\alpha / 2}$. Suppose that $b$ is a locally integrable function on $\mathbb{R}^{d}$ and the commutator generated by $b$ and $\mathscr{I}_{\alpha}$ is defined by $\left[b . \mathscr{I}_{\alpha}\right] f(x)=b(x) \cdot \mathscr{I}_{\alpha} f(x)-\mathscr{I}_{\alpha}(b f)(x)$. In this paper, we first introduce some kinds of weighted Morrey spaces related to certain nonnegative potentials belonging to the reverse Hölder class $\mathrm{RH}_{s}$ with $s \geq d / 2$. Then, we will establish the boundedness properties of the fractional integrals $\mathscr{I}_{\alpha}$ on these new spaces. Furthermore, weighted strong-type estimate for the corresponding commutator $\left[b, \mathscr{I}_{\alpha}\right]$ in the framework of Morrey space is also obtained. The classes of weights, the classes of symbol functions, as well as weighted Morrey spaces discussed in this paper are larger than $A_{p, q}, \operatorname{BMO}\left(\mathbb{R}^{d}\right)$, and $L^{p, \kappa}(\mu, \nu)$ corresponding to the classical case (that is $V \equiv 0$ ).

\section{Introduction}

1.1. Critical Radius Function $\rho(x)$. Let $d \geq 3$ be a positive integer and $\mathbb{R}^{d}$ be the $d$-dimensional Euclidean space, and let $V: \mathbb{R}^{d} \longrightarrow \mathbb{R}, d \geq 3$, be a nonnegative locally integrable function that belongs to the reverse Hölder class $\mathrm{RH}_{s}$ for some exponent $1<s<\infty$; i.e., there exists a positive constant $C>0$ such that the following reverse Hölder inequality

$$
\left(\frac{1}{|B|} \int_{B} V(y)^{s} \mathrm{~d} y\right)^{1 / s} \leq C\left(\frac{1}{|B|} \int_{B} V(y) \mathrm{d} y\right)
$$

holds for every ball $B$ in $\mathbb{R}^{d}$. For given $V \in \mathrm{RH}_{s}$ with $s \geq d / 2$, we introduce the critical radius function $\rho(x)=\rho(x ; V)$ which is given by

$$
\rho(x):=\sup \left\{r>0: \frac{1}{r^{d-2}} \int_{B(x, r)} V(y) \mathrm{d} y \leq 1\right\}, \quad x \in \mathbb{R}^{d},
$$

where $B(x, r)$ denotes the open ball centered at $x$ and with radius $r$. It is well known that this auxiliary function satisfies $0<\rho(x)<\infty$ for any $x \in \mathbb{R}^{d}$ under the above condition on $V$ (see [1]). We need the following known result concerning the critical radius function (2).

Lemma 1 (see [1]). If $V \in R H_{s}$ with $s \geq d / 2$, then there exist two constants $C_{0} \geq 1$ and $N_{0}>0$ such that, for all $x$ and $y$ in $\mathbb{R}^{d}$

$$
\frac{1}{C_{0}}\left(1+\frac{|x-y|}{\rho(x)}\right)^{-N_{0}} \leq \frac{\rho(y)}{\rho(x)} \leq C_{0}\left(1+\frac{|x-y|}{\rho(x)}\right)^{N_{0} /\left(N_{0}+1\right)} .
$$

As a straightforward consequence of (3), we can see that, for each integer $k \geq 1$, the following estimate

$$
1+\frac{2^{k} r}{\rho(y)} \geq \frac{1}{C_{0}}\left(1+\frac{r}{\rho(x)}\right)^{-\left(N_{0} /\left(N_{0}+1\right)\right)}\left(1+\frac{2^{k} r}{\rho(x)}\right),
$$

is valid for any $y \in B(x, r)$ with $x \in \mathbb{R}^{d}$ and $r>0$, and $C_{0}$ is defined in (3). 
1.2. Fractional Integrals Associated with Schrödinger Operators. Let $V \in \mathrm{RH}_{s}$ with $s \geq d / 2$. For such a potential $V$, we consider the Schrödinger differential operator on $\mathbb{R}^{d}$, $d \geq 3$,

$$
\mathscr{L}:=-\Delta+V
$$

and its associated semigroup

$$
\mathscr{T}_{t} f(x):=e^{-t \mathscr{L}} f(x)=\int_{\mathbb{R}^{d}} p_{t}(x, y) f(y) \mathrm{d} y, \quad t>0,
$$

where $p_{t}(x, y)$ denotes the kernel of the operator $e^{-t \mathscr{L}}, t>0$. From the Feynman-Kac formula, it is well known that

$$
\left|p_{t}(x, y)\right| \leq h_{t}(x-y), \quad t>0,
$$

where $h_{t}$ is the classical heat kernel; i.e.,

$$
h_{t}(x-y):=(4 \pi t)^{-d / 2} \exp \left(-\frac{|x-y|^{2}}{4 t}\right) \text {. }
$$

Moreover, this estimate (7) can be improved when $V$ belongs to the reverse Hölder class $R H_{s}$ for some $s \geq d / 2$ (see $[2,3]$, for instance). The auxiliary function $\rho(x)$ arises naturally in this context.

Proposition 1. Let $V \in R H_{s}$ with $s \geq d / 2$. For every positive integer $N \geq 1$, there exists a positive constant $C_{N}>0$ such that, for all $x$ and $y$ in $\mathbb{R}^{d}$,

$$
\left|p_{t}(x, y)\right| \leq C_{N} \cdot t^{-d / 2} \exp \left(-\frac{|x-y|^{2}}{5 t}\right)\left(1+\frac{\sqrt{t}}{\rho(x)}+\frac{\sqrt{t}}{\rho(y)}\right)^{-N}, \quad t>0 .
$$

For given $0<\alpha<d$, the $\mathscr{L}$-Riesz potential or $\mathscr{L}$-fractional integral operator is defined by

$$
\begin{aligned}
\mathscr{I}_{\alpha} f(x) & :=\mathscr{L}^{-\alpha / 2} f(x) \\
& =\int_{0}^{\infty} e^{-t \mathscr{L}} f(x) t^{\alpha / 2-1} \mathrm{~d} t .
\end{aligned}
$$

In this work, we shall be interested in the behavior of the $\mathscr{L}$-fractional integral operator $\mathscr{I}_{\alpha}=\mathscr{L}^{-\alpha / 2}$.

1.3. $A_{p}^{\rho, \infty}$ and $A_{p, q}^{\rho, \infty}$ Weights. A weight will always mean a nonnegative function which is locally integrable on $\mathbb{R}^{d}$. Given a Lebesgue measurable set $E$ and a weight $w,|E|$ will denote the Lebesgue measure of $E$ and

$$
w(E)=\int_{E} w(x) \mathrm{d} x
$$

Given $B=B\left(x_{0}, r\right)$ and $t>0$, we will write $t B$ for the $t$ dilate ball, which is the ball with the same center $x_{0}$ and radius $t r$. As in [2] (see also [4, 5]), we say that a weight $w$ belongs to the class $A_{p}^{\rho, \theta}$ for $1<p<\infty$ and $0<\theta<\infty$, if there is a positive constant $C>0$ such that, for all balls $B=B\left(x_{0}, r\right) \subset \mathbb{R}^{d}$ with $x_{0} \in \mathbb{R}^{d}$ and $r>0$,

$$
\left(\frac{1}{|B|} \int_{B} w(x) \mathrm{d} x\right)^{1 / p}\left(\frac{1}{|B|} \int_{B} w(x)^{-p^{\prime} / p} \mathrm{~d} x\right)^{1 / p^{\prime}} \leq C \cdot\left(1+\frac{r}{\rho\left(x_{0}\right)}\right)^{\theta},
$$

where $p^{\prime}$ is the dual exponent of $p$ such that $(1 / p)+\left(1 / p^{\prime}\right)=1$. For $p=1$ and $0<\theta<\infty$, we also say that a weight $w$ belongs to the class $A_{1}^{\rho, \theta}$, if there is a positive constant $C>0$ such that, for all balls $B=B\left(x_{0}, r\right) \subset \mathbb{R}^{d}$ with $x_{0} \in \mathbb{R}^{d}$ and $r>0$,

$$
\frac{1}{|B|} \int_{B} w(x) \mathrm{d} x \leq C \cdot\left(1+\frac{r}{\rho\left(x_{0}\right)}\right)^{\theta} \underset{x \in B}{\operatorname{essinf}} w(x) .
$$

For given $1 \leq p<\infty$, we define

$$
A_{p}^{\rho, \infty}:=\bigcup_{\theta>0} A_{p}^{\rho, \theta} .
$$

For any given $\theta>0$, let us introduce the maximal operator which is given in terms of the critical radius function (2):

$$
M_{\rho, \theta} f(x):=\sup _{r>0}\left(1+\frac{r}{\rho(x)}\right)^{-\theta} \frac{1}{|B(x, r)|} \int_{B(x, r)}|f(y)| \mathrm{d} y, \quad x \in \mathbb{R}^{d} .
$$

Observe that a weight $w$ belongs to the class $A_{1}^{\rho, \infty}$ if and only if there exists a positive number $\theta>0$ such that $M_{\rho, \theta}(w)(x) \leq C w(x)$, for a.e. $x \in \mathbb{R}^{d}$, where the constant $C>0$ is independent of $w$. Since

$$
1 \leq\left(1+\frac{r}{\rho\left(x_{0}\right)}\right)^{\theta_{1}} \leq\left(1+\frac{r}{\rho\left(x_{0}\right)}\right)^{\theta_{2}},
$$

for $0<\theta_{1}<\theta_{2}<\infty$, then for given $p$ with $1 \leq p<\infty$, one has

$$
A_{p} \subset A_{p}^{\rho, \theta_{1}} \subset A_{p}^{\rho, \theta_{2}}
$$

where $A_{p}$ denotes the classical Muckenhoupt's class (see [6], Chapter 7) and hence $A_{p} \subset A_{p}^{\rho, \infty}$. In addition, for some fixed $\theta>0$ (see [7]),

$$
A_{1}^{\rho, \theta} \subset A_{p_{1}}^{\rho, \theta} \subset A_{p_{2}}^{\rho, \theta}
$$

whenever $1 \leq p_{1}<p_{2}<\infty$. Obviously, for any fixed $\theta>0$,

$$
1 \leq\left(1+\frac{2 r}{\rho\left(x_{0}\right)}\right)^{\theta} \leq 2^{\theta}\left(1+\frac{r}{\rho\left(x_{0}\right)}\right)^{\theta} .
$$

To establish weighted norm inequalities for fractional integrals, we need to introduce another weight class $A_{p, q}^{\rho, \infty}$. As in [7], we say that a weight $w$ satisfies the condition $A_{p, q}^{\rho, \theta}$ for $1<p<q<\infty$ and $0<\theta<\infty$, if there exists a positive constant $C>0$ such that, for any ball $B=B\left(x_{0}, r\right) \subset \mathbb{R}^{d}$ with $x_{0} \in \mathbb{R}^{d}$ and $r>0$,

$$
\left(\frac{1}{|B|} \int_{B} w(x)^{q} \mathrm{~d} x\right)^{1 / q}\left(\frac{1}{|B|} \int_{B} w(x)^{-p^{\prime}} \mathrm{d} x\right)^{1 / p^{\prime}} \leq C \cdot\left(1+\frac{r}{\rho\left(x_{0}\right)}\right)^{\theta},
$$

where $p^{\prime}=p /(p-1)$. We also say that a weight $w$ satisfies the condition $A_{1, q}^{\rho, \theta}$ for $1<q<\infty$ and $0<\theta<\infty$, if there exists a positive constant $C>0$ such that, for any ball $B=B\left(x_{0}, r\right) \subset \mathbb{R}^{d}$ with $x_{0} \in \mathbb{R}^{d}$ and $r>0$, 


$$
\left(\frac{1}{|B|} \int_{B} w(x)^{q} \mathrm{~d} x\right)^{1 / q} \leq C \cdot\left(1+\frac{r}{\rho\left(x_{0}\right)}\right)^{\theta} \underset{x \in B}{\operatorname{essinf}} w(x) .
$$

Similarly, for given $p, q$ with $1 \leq p<q<\infty$, by (16), one has

$$
A_{p, q} \subset A_{p, q}^{\rho, \theta_{1}} \subset A_{p, q}^{\rho, \theta_{2}},
$$

whenever $0<\theta_{1}<\theta_{2}<\infty$. Here, $A_{p, q}$ denotes the classical Muckenhoupt-Wheeden's class (see [8]). We also define

$$
A_{p, q}^{\rho, \infty}:=\bigcup_{\theta>0} A_{p, q}^{\rho, \theta}, \quad 1 \leq p<q<\infty .
$$

So, we have $A_{p, q} \subset A_{p, q}^{\rho, \infty}$. The following results (Lemmas 2-5) are extensions of well-known properties of $A_{p}$ and $A_{p, q}$ weights. We first present an important property of the classes of weights in $A_{p}^{\rho, \theta}$ with $1 \leq p<\infty$, which was given by Bongioanni, Harboure, and Salinas in Lemma 5 of [2].

Lemma 2 (see [2]). If $w \in A_{p}^{\rho, \theta}$ with $0<\theta<\infty$ and $1 \leq p<\infty$, then there exist positive constants $\epsilon, \eta>0$, and $C>0$ such that

$$
\left(\frac{1}{|B|} \int_{B} w(x)^{1+\varepsilon} \mathrm{d} x\right)^{1 / 1+\varepsilon} \leq C\left(\frac{1}{|B|} \int_{B} w(x) \mathrm{d} x\right)\left(1+\frac{r}{\rho\left(x_{0}\right)}\right)^{\eta},
$$

for every ball $B=B\left(x_{0}, r\right)$ in $\mathbb{R}^{d}$.

As a direct consequence of Lemma 2, we have the following result.

Lemma 3. If $w \in A_{p}^{\rho, \theta}$ with $0<\theta<\infty$ and $1 \leq p<\infty$, then there exist two positive numbers $\delta>0$ and $\eta>0$ such that

$$
\frac{w(E)}{w(B)} \leq C\left(\frac{|E|}{|B|}\right)^{\delta}\left(1+\frac{r}{\rho\left(x_{0}\right)}\right)^{\eta},
$$

for any measurable subset $E$ of a ball $B=B\left(x_{0}, r\right)$, where $C>0$ is a constant which does not depend on $E$ and $B$.

Proof. For any given ball $B=B\left(x_{0}, r\right)$ with $x_{0} \in \mathbb{R}^{d}$ and $r>0$, suppose that $E \subset B$, then by Hölder's inequality with exponent $1+\epsilon$ and (24), we can deduce that

$$
\begin{aligned}
w(E) & =\int_{B} \chi_{E}(x) \cdot w(x) \mathrm{d} x \\
& \leq\left(\int_{B} w(x)^{1+\varepsilon} \mathrm{d} x\right)^{1 /(1+\varepsilon)}\left(\int_{B} \chi_{E}(x)^{(1+\varepsilon) / \varepsilon} \mathrm{d} x\right)^{\varepsilon / 1+\varepsilon} \\
& \leq C|B|^{1 /(1+\varepsilon)}\left(\frac{1}{|B|} \int_{B} w(x) \mathrm{d} x\right)\left(1+\frac{r}{\rho\left(x_{0}\right)}\right)^{\eta}|E|^{\varepsilon /(1+\varepsilon)} \\
& =C\left(\frac{|E|}{|B|}\right)^{\varepsilon /(1+\varepsilon)}\left(1+\frac{r}{\rho\left(x_{0}\right)}\right)^{\eta} w(B) .
\end{aligned}
$$

This gives (25) with $\delta=\epsilon /(1+\epsilon)$. Here and in the sequel, the characteristic function of $E$ is denoted by $\chi_{E}$.
In view of Lemma 2, we now define the reverse Höldertype class $\mathrm{RH}_{a}^{\rho, \theta}$ that is given in terms of the critical radius function (2). We say that $w \in \mathrm{RH}_{q}^{\rho, \theta}$ for some $1<q<\infty$ and $0<\theta<\infty$, if there exists a positive constant $C>0$ such that the following reverse Hölder-type inequality

$$
\left(\frac{1}{|B|} \int_{B} w(x)^{q} \mathrm{~d} x\right)^{1 / q} \leq C\left(\frac{1}{|B|} \int_{B} w(x) \mathrm{d} x\right)\left(1+\frac{r}{\rho\left(x_{0}\right)}\right)^{\theta}
$$

holds for every ball $B=B\left(x_{0}, r\right)$ in $\mathbb{R}^{d}$. The class $R H_{q}^{\rho, \infty}$ is defined as

$$
\mathrm{RH}_{q}^{\rho, \infty}:=\bigcup_{\theta>0} \mathrm{RH}_{q}^{\rho, \theta}, \quad 1<q<\infty .
$$

Clearly, one has $R H_{q} \subset R H_{q}^{\rho, \infty}$.

Let $1<q<\infty$ and $A_{1}^{q}=\left\{w: w^{q} \in A_{1}\right\}$. It is known that, for the classical case (see [9]),

$$
A_{1}^{q}=A_{1} \cap \mathrm{RH}_{q} .
$$

Lemma 4. If $1<q<\infty$ and $0<\theta<\infty$, then $w^{q} \in A_{1}^{\rho, \theta}$ implies that $w \in A_{1}^{\rho, \theta / q} \cap R H_{q}^{\rho, \theta / q}$.

Proof. The conclusion $w \in A_{1}^{\rho, \theta / q}$ follows easily by Hölder's inequality and the definition of $A_{1}^{\rho, \theta}$. Indeed, for any given ball $B=B\left(x_{0}, r\right)$ with $x_{0} \in \mathbb{R}^{d}$ and $r>0$,

$$
\begin{aligned}
\frac{1}{|B|} \int_{B} w(x) \mathrm{d} x & \leq\left(\frac{1}{|B|} \int_{B} w^{q}(x) \mathrm{d} x\right)^{1 / q} \\
& \leq C \cdot\left(1+\frac{r}{\rho\left(x_{0}\right)}\right)^{\theta / q}\left(\underset{x \in B}{\operatorname{essinf}} w^{q}(x)\right)^{1 / q} \\
& =C \cdot\left(1+\frac{r}{\rho\left(x_{0}\right)}\right)^{\theta / q} \underset{x \in B}{\operatorname{essinf}} w(x) .
\end{aligned}
$$

On the other hand, fix a ball $B=B\left(x_{0}, r\right) \subset \mathbb{R}^{d}$ and take $y \in B$. Let $E$ be a ball centered at $y_{0}$ and with radius $h$ which contains $y$. By picking $h$ small enough so that $E \subset B$, then by the condition $w^{q} \in A_{1}^{\rho, \theta}$, we can deduce that

$$
\begin{aligned}
\frac{1}{|B|} \int_{B} \chi_{E}(x) \mathrm{d} x & \leq \frac{C}{w^{q}(B)} \cdot \underset{x \in B}{\operatorname{essinf}} w^{q}(x)\left(\int_{B} \chi_{E}(x) \mathrm{d} x\right)\left(1+\frac{r}{\rho\left(x_{0}\right)}\right)^{\theta} \\
& \leq \frac{C}{w^{q}(B)}\left(\int_{B} \chi_{E}(x) \cdot w^{q}(x) \mathrm{d} x\right)\left(1+\frac{r}{\rho\left(x_{0}\right)}\right)^{\theta},
\end{aligned}
$$

which is equivalent to

$$
\frac{w^{q}(B)}{|B|} \leq C \cdot \frac{w^{q}(E)}{|E|}\left(1+\frac{r}{\rho\left(x_{0}\right)}\right)^{\theta} .
$$

Since this holds for all $E \subset B$ and $y \in E$, then by taking $h \longrightarrow 0^{+}$and using Lebesgue differentiation theorem, 


$$
\frac{w^{q}(B)}{|B|} \leq C \cdot w^{q}(y)\left(1+\frac{r}{\rho\left(x_{0}\right)}\right)^{\theta}
$$

Thus, by raising both sides to the power $1 / q$ and integrating over $B$, we get

$$
\left(\frac{1}{|B|} \int_{B} w(y)^{q} \mathrm{~d} y\right)^{1 / q} \leq C\left(\frac{1}{|B|} \int_{B} w(y) \mathrm{d} y\right)\left(1+\frac{r}{\rho\left(x_{0}\right)}\right)^{\theta / q}
$$

This amounts to $w \in \mathrm{RH}_{q}^{\rho, \theta / q}$.

A subtle interplay between these two classes of weights, $A_{p}^{\rho, \infty}$ and $A_{p, q}^{\rho, \infty}$, is expressed by the following lemma:

Lemma 5. Suppose that $1 \leq p<q<\infty$. Then, the following statements are true:

(i) If $p>1$ and $0<\theta<\infty$, then $w \in A_{p, q}^{\rho, \theta}$ implies that $w^{q} \in A_{t}^{\rho, \theta \cdot\left(1 /\left((1 / q)+\left(1 / p^{\prime}\right)\right)\right)}$ with $t=1+q / p^{\prime} \quad$ and $w^{-p^{\prime}} \in A_{t^{\prime}}^{\rho, \theta \cdot\left(1 /\left((1 / q)+\left(1 / p^{\prime}\right)\right)\right)}$ with $t^{\prime}=1+p^{\prime} / q$;

(ii) If $p=1$ and $0<\theta<\infty$, then $w \in A_{1, q}^{\rho, \theta}$ implies that $w^{q} \in A_{1}^{\rho, \theta \cdot q}$.

Proof. In fact, when $t=1+q / p^{\prime}$, then a simple computation shows that

$$
\begin{aligned}
\frac{1}{t} & =\frac{1}{q} \cdot \frac{1}{(1 / q)+\left(1 / p^{\prime}\right)} \\
\frac{1}{t^{\prime}} & =\frac{t-1}{t}=\frac{1}{p^{\prime}} \cdot \frac{1}{(1 / q)+\left(1 / p^{\prime}\right)} \\
q \cdot\left(-\frac{t^{\prime}}{t}\right) & =-q \cdot \frac{1}{t-1}=-p^{\prime} .
\end{aligned}
$$
have

If $w \in A_{p, q}^{\rho, \theta}$ with $1<p<q<\infty$ and $0<\theta<\infty$, then we

$$
\begin{aligned}
& \left(\frac{1}{|B|} \int_{B} w^{q}(x) \mathrm{d} x\right)^{1 / t}\left(\frac{1}{|B|} \int_{B} w^{q}(x)^{-t^{\prime} / t} \mathrm{~d} x\right)^{1 / t^{\prime}} \\
& \quad=\left[\left(\frac{1}{|B|} \int_{B} w(x)^{q} \mathrm{~d} x\right)^{1 / q}\left(\frac{1}{|B|} \int_{B} w(x)^{-p^{\prime}} \mathrm{d} x\right)^{1 / p^{\prime}}\right]^{1 /\left((1 / q)+\left(1 / p^{\prime}\right)\right)} \\
& \leq C \cdot\left(1+\frac{r}{\rho\left(x_{0}\right)}\right)^{\theta \cdot\left(1 /\left((1 / q)+\left(1 / p^{\prime}\right)\right)\right)},
\end{aligned}
$$

which means that $w^{q} \in A_{t}^{\rho, \theta \cdot\left(1 /\left((1 / q)+\left(1 / p^{\prime}\right)\right)\right)}$ with $t=1+q / p^{\prime}$. Here and in the sequel, for any positive number $\gamma>0$, we denote $w^{\gamma}(x)=w(x)^{\gamma}$ by convention. Analogously, it can be easily shown that $w^{-} p^{\prime} \in A_{t^{\prime}}^{\rho, \theta \cdot\left(1 /\left((1 / q)+\left(1 / p^{\prime}\right)\right)\right)}$ with $t^{\prime}=1+p^{\prime} / q$. On the other hand, if $w \in A_{1, q}^{\rho, \theta}$ with $1<q<\infty$ and $0<\theta<\infty$, then we have

$$
\begin{aligned}
\frac{1}{|B|} \int_{B} w^{q}(x) \mathrm{d} x & \leq C \cdot\left(1+\frac{r}{\rho\left(x_{0}\right)}\right)^{\theta \cdot q}(\underset{x \in B}{\operatorname{essinf}} w(x))^{q} \\
& =C \cdot\left(1+\frac{r}{\rho\left(x_{0}\right)}\right)^{\theta \cdot q} \underset{x \in B}{\operatorname{essinf}} w^{q}(x),
\end{aligned}
$$

which means that $w^{q} \in A_{1}^{\rho, \theta \cdot q}$.

Given a weight $w$ on $\mathbb{R}^{d}$, as usual, the weighted Lebesgue space $L^{p}(w)$ for $1 \leq p<\infty$ is defined to be the set of all functions $f$ such that

$$
\|f\|_{L^{p}(w)}:=\left(\int_{\mathbb{R}^{d}}|f(x)|^{p} w(x) \mathrm{d} x\right)^{1 / p}<\infty .
$$

We also denote by $W L^{p}(w)$ the weighted weak Lebesgue space consisting of all measurable functions $f$ for which

$$
\|f\|_{W L^{p}(w)}:=\sup _{\lambda>0} \lambda \cdot\left[w\left(\left\{x \in \mathbb{R}^{d}:|f(x)|>\lambda\right\}\right)\right]^{1 / p}<\infty .
$$

Note that if $\mathscr{L}=-\Delta$ is the Laplacian on $\mathbb{R}^{d}$, then $\mathscr{L}^{-\alpha / 2}$ is the classical fractional integral operator $I_{\alpha}=(-\Delta)^{-\alpha / 2}$ of order $\alpha$. Let $0<\alpha<d$ and $1 \leq p<d / \alpha$. Define $1<q<\infty$ by the relation $1 / q=1 / p-\alpha / d$. It is well known that when $p>1$ and $w \in A_{p, q}, I_{\alpha}$ is bounded from $L^{p}\left(w^{p}\right)$ into $L^{q}\left(w^{q}\right)$. When $p=1$ and $w \in A_{1, q}, I_{\alpha}$ is bounded from $L^{1}(w)$ into $W L^{q}\left(w^{q}\right)$ (see [8]). Recently, Bongioanni et al. ([2], Theorem 4) established the weighted boundedness for fractional integral operators $\mathscr{I}_{\alpha}$ associated with Schrödinger operators defined in (10). They showed that the same estimates also hold for weights in the class $A_{p, q}^{\rho, \infty}$, which is larger than Muckenhoupt-Wheeden's class (another proof was later given by Tang in Theorem 3.8 of [7]). Their results can be summarized as follows:

Theorem 1 (see [2]). Let $0<\alpha<d, \quad 1<p<d / \alpha$, $1 / q=1 / p-\alpha / d$, and $w \in A_{p, q}^{\rho, \infty}$. If $V \in R H_{s}$ with $s \geq d / 2$, then the $\mathscr{L}$-fractional integral operator $\mathscr{I}_{\alpha}$ is bounded from $L^{p}\left(w^{p}\right)$ into $L^{q}\left(w^{q}\right)$.

Theorem 2 (see [2]). Let $0<\alpha<d, p=1, q=d /(d-\alpha)$, and $w \in A_{1, q}^{\rho, \infty}$. If $V \in R H_{s}$ with $s \geq d / 2$, then the $\mathscr{L}$-fractional integral operator $\mathscr{I}_{\alpha}$ is bounded from $L^{1}(w)$ into $W L^{q}\left(w^{q}\right)$.

1.4. The $B M O_{\rho, \infty}\left(\mathbb{R}^{d}\right)$ Space. For a locally integrable function $b$ on $\mathbb{R}^{d}$ (usually called the symbol), we will also consider the commutator operator

$$
\left[b, \mathscr{I}_{\alpha}\right] f(x):=b(x) \cdot \mathscr{I}_{\alpha} f(x)-\mathscr{I}_{\alpha}(b f)(x), \quad x \in \mathbb{R}^{d} .
$$

Recently, Bongioanni et al. [5] introduced a kind of new spaces $\mathrm{BMO}_{\rho, \infty}\left(\mathbb{R}^{d}\right)$ defined by

$$
\mathrm{BMO}_{\rho, \infty}\left(\mathbb{R}^{d}\right):=\bigcup_{\theta>0} \mathrm{BMO}_{\rho, \theta}\left(\mathbb{R}^{d}\right)
$$

where for $0<\theta<\infty$, the space $\mathrm{BMO}_{\rho, \theta}\left(\mathbb{R}^{d}\right)$ is defined to be the set of all locally integrable functions $b$ satisfying 


$$
\frac{1}{\left|B\left(x_{0}, r\right)\right|} \int_{B\left(x_{0}, r\right)}\left|b(x)-b_{B\left(x_{0}, r\right) \mid}\right| \mathrm{d} x \leq C \cdot\left(1+\frac{r}{\rho\left(x_{0}\right)}\right)^{\theta},
$$

for all balls $B\left(x_{0}, r\right)$ with $x_{0} \in \mathbb{R}^{d}$ and $r>0$ and $b_{B\left(x_{0}, r\right)}$ denotes the mean value of $b$ on $B\left(x_{0}, r\right)$; that is,

$$
b_{B\left(x_{0}, r\right)}:=\frac{1}{\left|B\left(x_{0}, r\right)\right|} \int_{B\left(x_{0}, r\right)} b(y) \mathrm{d} y .
$$

A norm for $b \in \mathrm{BMO}_{\rho, \theta}\left(\mathbb{R}^{d}\right)$, denoted by $\|b\|_{\mathrm{BMO}_{\rho, \theta}}$, is given by the infimum of the constants satisfying $(42)$, or equivalently

$$
\begin{aligned}
\|b\|_{\mathrm{BMO}_{\rho, \theta}:=} & \sup _{B\left(x_{0}, r\right)}\left(1+\frac{r}{\rho\left(x_{0}\right)}\right)^{-\theta} \\
& \cdot\left(\frac{1}{\left|B\left(x_{0}, r\right)\right|} \int_{B\left(x_{0}, r\right)}\left|b(x)-b_{B\left(x_{0}, r\right) \mid}\right| \mathrm{d} x\right),
\end{aligned}
$$

where the supremum is taken over all balls $B\left(x_{0}, r\right)$ with $x_{0} \in \mathbb{R}^{d}$ and $r>0$. With the above definition in mind, one has

$$
\operatorname{BMO}\left(\mathbb{R}^{d}\right) \subset \mathrm{BMO}_{\rho, \theta_{1}}\left(\mathbb{R}^{d}\right) \subset \mathrm{BMO}_{\rho, \theta_{2}}\left(\mathbb{R}^{d}\right),
$$

for $0<\theta_{1}<\theta_{2}<\infty$ by virtue of (16), and hence, $\operatorname{BMO}\left(\mathbb{R}^{d}\right) \subset$ $\mathrm{BMO}_{\rho, \infty}\left(\mathbb{R}^{d}\right)$. Moreover, the classical BMO space [10] is properly contained in $\mathrm{BMO}_{\rho, \infty}\left(\mathbb{R}^{d}\right)$ (see $[4,5]$ for more examples). We need the following key result for the space $\mathrm{BMO}_{\rho, \theta}\left(\mathbb{R}^{d}\right)$, which was proved by Tang in Proposition 4.2 of [7].

Proposition 2 (see [7]). Let $b \in B M O_{\rho, \theta}\left(\mathbb{R}^{d}\right)$ with $0<\theta<\infty$. Then, there exist two positive constants $C_{1}$ and $C_{2}$ such that, for any given ball $B\left(x_{0}, r\right)$ in $\mathbb{R}^{d}$ and for any $\lambda>0$, we have

$$
\begin{aligned}
& \mid\left\{x \in B\left(x_{0}, r\right): \mid b(x)-b_{\left.B\left(x_{0}, r\right) \mid>\lambda\right\} \mid}\right. \\
& \quad \leq C_{1}\left|B\left(x_{0}, r\right)\right| \exp \left[-\left(1+\frac{r}{\rho\left(x_{0}\right)}\right)^{-\theta^{*}} \frac{C_{2} \lambda}{\|b\|_{\mathrm{BMO}_{\rho, \theta}}}\right],
\end{aligned}
$$

where $\theta^{*}=\left(N_{0}+1\right) \theta$ and $N_{0}$ is the constant appearing in Lemma 1.

As a consequence of Proposition 2 and Lemma 3, we have the following result:

Proposition 3. Let $b \in B M O_{\rho, \theta}\left(\mathbb{R}^{d}\right)$ with $0<\theta<\infty$ and $w \in A_{p}^{p, \infty}$ with $1 \leq p<\infty$. Then, there exist positive constants $C_{1}, C_{2}$ and $\eta>0$ such that, for any given ball $B\left(x_{0}, r\right)$ in $\mathbb{R}^{d}$ and for any $\lambda>0$, we have

$$
\begin{aligned}
& w\left(\left\{x \in B\left(x_{0}, r\right):\left|b(x)-b_{B\left(x_{0}, r\right)}\right|>\lambda\right\}\right) \\
& \quad \leq C_{1} w\left(B\left(x_{0}, r\right)\right) \exp \left[-\left(1+\frac{r}{\rho\left(x_{0}\right)}\right)^{-\theta^{*}} \frac{C_{2} \lambda}{\|b\|_{\mathrm{BMO}_{\rho, \theta}}}\right]\left(1+\frac{r}{\rho\left(x_{0}\right)}\right)^{\eta},
\end{aligned}
$$

where $\theta^{*}=\left(N_{0}+1\right) \theta$ and $N_{0}$ is the constant appearing in Lemma 1.

Notice that if $\mathscr{L}=-\Delta$ is the Laplacian operator on $\mathbb{R}^{d}$, then $\left[b, \mathscr{I}_{\alpha}\right]$ is just the commutator $\left[b, I_{\alpha}\right]$ of the classical fractional integrals. It is well known that when $b \in \mathrm{BMO}\left(\mathbb{R}^{d}\right)$, the commutator $\left[b, I_{\alpha}\right]$ is bounded from $L^{p}\left(w^{p}\right)(1<p<d / \alpha)$ into $L^{q}\left(w^{q}\right)$ whenever $1 / q=(1 / p)-$ $(\alpha / d)$ and $w \in A_{p, q}$ (see [12]). This corresponds to the norm inequalities satisfied by $I_{\alpha}$. In Theorem 4.4 of [7], Tang obtained weighted strong-type estimate for the commutator $\left[b, \mathscr{I}_{\alpha}\right]$ of fractional integrals associated with Schrödinger operators, when $b$ in a larger space than $\operatorname{BMO}\left(\mathbb{R}^{d}\right)$, that is the space $\mathrm{BMO}_{\rho, \infty}\left(\mathbb{R}^{d}\right)$. More precisely, he gave the following weighted result (see also [11], Theorem 3.5).

Theorem 3 (see [7]). Let $0<\alpha<d, \quad 1<p<d / \alpha$, $1 / q=(1 / p)-(\alpha / d)$, and $w \in A_{p, q}^{\rho, \infty}$. If $V \in R H_{s}$ with $s \geq d / 2$, then the commutator operator $\left[b, \mathscr{I}_{\alpha}\right]$ is bounded from $L^{p}\left(w^{p}\right)$ into $L^{q}\left(w^{q}\right)$, whenever $b$ belongs to $B M O_{\rho, \infty}\left(\mathbb{R}^{d}\right)$.

In this paper, firstly, we will define several classes of weighted Morrey spaces related to certain nonnegative potentials satisfying appropriate reverse Hölder inequality. Secondly, we establish weighted estimates of fractional integrals $\mathscr{I}_{\alpha}$ associated with $\mathscr{L}$ on these new spaces. Finally, we also study the boundedness property for the commutators $\left[b, \mathscr{I}_{\alpha}\right]$ of fractional integrals with the new BMO functions defined above.

Throughout this paper, $C$ denotes a positive constant not necessarily the same at each occurrence, and a subscript is added when we wish to make clear its dependence on the parameter in the subscript. We also use $a \approx b$ to denote the equivalence of $a$ and $b$; that is, there exist two positive constants $C_{1}$ and $C_{2}$ independent of $a$ and $b$ such that $C_{1} a \leq b \leq C_{2} a$.

\section{Main Results}

In this section, we introduce some types of weighted Morrey spaces related to the nonnegative potential $V$ and then give our main results.

Definition 1. Let $1 \leq p<\infty, 0 \leq \kappa<1$, and $\mu$, $\nu$ be two weights on $\mathbb{R}^{d}$. For given $0<\theta<\infty$, the weighted Morrey space $L_{\rho, \theta}^{p, \kappa}(\mu, \nu)$ is defined to be the set of all $p$-locally integrable functions $f$ on $\mathbb{R}^{d}$ such that

$$
\left(\frac{1}{\nu(B)^{\kappa}} \int_{B}|f(x)|^{p} \mu(x) \mathrm{d} x\right)^{1 / p} \leq C \cdot\left(1+\frac{r}{\rho\left(x_{0}\right)}\right)^{\theta},
$$

for every ball $B=B\left(x_{0}, r\right)$ in $\mathbb{R}^{d}$. A norm for $f \in L_{\rho, \theta}^{p, \kappa}(\mu, \nu)$, denoted by $\|f\|_{L_{p, \theta}^{p, \kappa}(\mu, \nu)}$, is given by the infimum of the constants in (48), or equivalently,

$$
\begin{aligned}
\|f\|_{L_{\rho, \theta}^{p, \kappa}(\mu, v)}:= & \sup _{B}\left(1+\frac{r}{\rho\left(x_{0}\right)}\right)^{-\theta} \\
& \cdot\left(\frac{1}{\nu(B)^{\kappa}} \int_{B}|f(x)|^{p} \mu(x) \mathrm{d} x\right)^{1 / p}<\infty,
\end{aligned}
$$


where the supremum is taken over all balls $B$ in $\mathbb{R}^{d}$ and $x_{0}$ and $r$ denote the center and radius of $B$, respectively. Define

$$
L_{\rho, \infty}^{p, \kappa}(\mu, \nu):=\bigcup_{\theta>0} L_{\rho, \theta}^{p, \kappa}(\mu, \nu) .
$$

For any given $f \in L_{\rho, \infty}^{p, \kappa}(\mu, \nu)$, let

$$
\theta^{*}:=\inf \left\{\theta>0: f \in L_{\rho, \theta}^{p, \kappa}(\mu, \nu)\right\} .
$$

Now, define

$$
\|f\|_{\star}=\|f\|_{L_{\rho, \infty}^{p, \kappa}(\mu, \nu)}:=\|f\|_{L_{\rho, \theta^{*}}^{p, \kappa}(\mu, \nu)} .
$$

It is easy to see that $\|\cdot\|_{\star}$ satisfies the axioms of a norm; i.e., for $f, g \in L_{\rho, \infty}^{p, \kappa}(\mu, \nu)$ and $\lambda \in \mathbb{R}$, we have

(i) $\|f\|_{\star} \geq 0$

(ii) $\|f\|_{\star}=0 \Longleftrightarrow f=0$

(iii) $\|\lambda f\|_{\star}=|\lambda|\|f\|_{\star}$

(iv) $\|f+g\|_{\star} \leq\|f\|_{\star}+\|g\|_{\star}$

If $\mu=\nu=w$, then we denote $L_{\rho, \theta}^{p, \kappa}(w)$ and $L_{\rho, \infty}^{p, \kappa}(w)$ for simplicity.

Note that this definition does not coincide with the one given in [13] (see also [14] for the unweighted case), but in view of the space $\mathrm{BMO}_{\rho, \infty}\left(\mathbb{R}^{d}\right)$ and the class $A_{p, q}^{\rho, \infty}$ defined above, it is more natural in our setting. Obviously, if we take $\theta=0$ or $V \equiv 0$, then this new space is just the familiar weighted Morrey space $L^{p, \kappa}(\mu, \nu)$ (or $L^{p, \kappa}(w)$ ), which was first introduced by Komori and Shirai in [15] (see also [16]).

Definition 2. Let $1 \leq p<\infty, 0 \leq \kappa<1$, and $w$ be a weight on $\mathbb{R}^{d}$. For given $0<\theta<\infty$, the weighted weak Morrey space $W L_{\rho, \theta}^{p, \kappa}(w)$ is defined to be the set of all measurable functions $f$ on $\mathbb{R}^{d}$ such that

$$
\begin{aligned}
& \frac{1}{w(B)^{\kappa / p}} \sup _{\lambda>0} \lambda \cdot[w(\{x \in B:|f(x)|>\lambda\})]^{1 / p} \\
& \quad \leq C \cdot\left(1+\frac{r}{\rho\left(x_{0}\right)}\right)^{\theta}
\end{aligned}
$$

for every ball $B=B\left(x_{0}, r\right)$ in $\mathbb{R}^{d}$, or equivalently,

$$
\begin{gathered}
\|f\|_{W L_{\rho, \theta}^{p, \kappa}(w)}:=\sup _{B}\left(1+\frac{r}{\rho\left(x_{0}\right)}\right)^{-\theta} \frac{1}{w(B)^{\kappa / p}} \sup _{\lambda>0} \lambda \\
\cdot[w(\{x \in B:|f(x)|>\lambda\})]^{1 / p}<\infty .
\end{gathered}
$$

Correspondingly, we define

$$
W L_{\rho, \infty}^{p, \kappa}(w):=\bigcup_{\theta>0} W L_{\rho, \theta}^{p, \kappa}(w) .
$$

For any given $f \in W L_{\rho, \infty}^{p, \kappa}(w)$, let

$$
\theta^{* *}:=\inf \left\{\theta>0: f \in W L_{\rho, \theta}^{p, \kappa}(w)\right\} .
$$

Similarly, we define

$$
\|f\|_{\star \star}=\|f\|_{W L_{\rho, \infty}^{p, \kappa}(w)}:=\|f\|_{W L_{\rho, \theta^{* *}}^{p, \kappa}(w)} .
$$

By the definition, we can easily show that $\|\cdot\|_{\star \star}$ satisfies the axioms of a (quasi) norm, and $W L_{\rho, \infty}^{p, \kappa}(w)$ is a (quasi) normed linear space. Clearly, if we take $\theta=0$ or $V \equiv 0$, then this space is just the weighted weak Morrey space $W L^{p, \kappa}(w)$ (see [17]). According to the above definitions, one has

$$
\left\{\begin{array}{l}
L^{p, \kappa}(\mu, \nu) \subset L_{\rho, \theta_{1}}^{p, \kappa}(\mu, \nu) \subset L_{\rho, \theta_{2}}^{p, \kappa}(\mu, \nu), \\
W L^{p, \kappa}(w) \subset W L_{\rho, \theta_{1}}^{p, \kappa}(w) \subset W L_{\rho, \theta_{2}}^{p, \kappa}(w),
\end{array}\right.
$$

for $0<\theta_{1}<\theta_{2}<\infty$. Hence, $L^{p, \kappa}(\mu, \nu) \subset L_{\rho, \infty}^{p, \kappa}(\mu, \nu)$ (in particular $\left.L^{p, \kappa}(w) \subset L_{\rho, \infty}^{p, \kappa}(w)\right)$ and $W L^{p, \kappa}(w) \subset W L_{\rho, \infty}^{p, \kappa}(w)$ for $(p, \kappa) \in[1, \infty) \times[0,1)$.

The space $L_{\rho, \theta}^{p, \kappa}(w)$ (or $\left.W L_{\rho, \theta}^{p, \kappa}(w)\right)$ could be viewed as an extension of weighted (or weak) Lebesgue space (when $\kappa=$ $\theta=0$ ). Naturally, one may ask the question whether the above conclusions (i.e., Theorems 1 and 2 as well as Theorem 3) still hold if replacing the weighted Lebesgue spaces by the weighted Morrey spaces. In this work, we will give a positive answer to this question. We now state our main results as follows.

For weighted strong-type and weak-type estimates of fractional integrals associated with $\mathscr{L}$ on $L_{\rho, \infty}^{p, \kappa}\left(w^{p}, w^{q}\right)$, where $w$ belongs to the new classes of weights, we have the following.

Theorem 4. Let $0<\alpha<d, 1<p<d / \alpha, 1 / q=(1 / p)-(\alpha / d)$, and $w \in A_{p, q}^{\rho, \infty}$. If $V \in R H_{s}$ with $s \geq d / 2$ and $0<\kappa<p / q$, then the $\mathscr{L}$-fractional integral operator $\mathscr{I}_{\alpha}$ is bounded from $L_{\rho, \infty}^{p, \kappa}\left(w^{p}, w^{q}\right)$ into $L_{\rho, \infty}^{q,(\kappa q) / p}\left(w^{q}\right)$.

Theorem 5. Let $0<\alpha<d, \quad p=1, \quad q=d /(d-\alpha)$, and $w \in A_{1, q}^{\rho, \infty}$. If $V \in R H_{s}$ with $s \geq d / 2$ and $0<\kappa<1 / q$, then the $\mathscr{L}$-fractional integral operator $\mathscr{I}_{\alpha}$ is bounded from $L_{\rho, \infty}^{1, \kappa}\left(w, w^{q}\right)$ into $W L_{\rho, \infty}^{q,(\kappa q)}\left(w^{q}\right)$.

Concerning the boundedness property of the commutators $\left[b, \mathscr{I}_{\alpha}\right]$ in the setting of weighted Morrey space, where $b$ is in the new BMO-type space, we shall prove the following.

Theorem 6. Let $0<\alpha<d, 1<p<d / \alpha, 1 / q=(1 / p)-(\alpha / d)$, and $w \in A_{p, q}^{\rho, \infty}$. If $V \in R H_{s}$ with $s \geq d / 2$ and $0<\kappa<p / q$, then the commutator operator $\left[b, \mathscr{I}_{\alpha}\right]$ is bounded from $L_{\rho, \infty}^{p, \kappa}\left(w^{p}, w^{q}\right)$ into $L_{\rho, \infty}^{q,(\kappa q) / p}\left(w^{q}\right)$, whenever $b \in B M O_{\rho, \infty}\left(\mathbb{R}^{d}\right)$.

Moreover, for the extreme case $\kappa=p / q$ of Theorem 4, we will show that the fractional integrals associated with $\mathscr{L}$ maps $L_{\rho, \infty}^{p, \kappa}\left(w^{p}, w^{q}\right)$ continuously into the new space $\mathrm{BMO}_{\rho, \infty}\left(\mathbb{R}^{d}\right)$. This result may be regarded as a supplement of Theorem 4 .

Theorem 7. Let $0<\alpha<d, 1<p<d / \alpha, 1 / q=(1 / p)-(\alpha / d)$, and $w \in A_{p, q}^{\rho, \infty}$. If $V \in R H_{s}$ with $s \geq d / 2$ and $\kappa=p / q$, then the $\mathscr{L}$-fractional integral operator $\mathscr{I}_{\alpha}$ is bounded from $L_{\rho, \infty}^{p, \kappa}\left(w^{p}, w^{q}\right)$ into $B M O_{\rho, \infty}\left(\mathbb{R}^{d}\right)$.

Remark 1. It is worth pointing out that, in the classical case when $V \equiv 0$, Theorems 4,5 , and 6 have been proved by Komori and Shirai in [15], while Theorem 7 has been shown by the author in [18]. 


\section{Proofs of Theorems 4 and 5}

In this section, we will prove the conclusions of Theorems 4 and 5. Let us remind that the $\mathscr{L}$-fractional integral operator of order $\alpha \in(0, d)$ can be written as

$$
\begin{aligned}
\mathscr{I}_{\alpha} f(x) & =\mathscr{L}^{-\alpha / 2} f(x)=\int_{0}^{\infty} e^{-t \mathscr{L}} f(x) t^{\alpha / 2-1} \mathrm{~d} t \\
& =\int_{\mathbb{R}^{d}}\left[\int_{0}^{\infty} p_{t}(x, y) t^{\alpha / 2-1} \mathrm{~d} t\right] f(y) \mathrm{d} y .
\end{aligned}
$$

The kernel of the fractional integral operator $\mathscr{F}_{\alpha}$ will be denoted by $\mathscr{K}_{\alpha}(x, y)$. Then, (see $\left.[19,20]\right)$

$$
\begin{aligned}
\mathscr{K}_{\alpha}(x, y) & =\int_{0}^{\infty} p_{t}(x, y) t^{\alpha / 2-1} \mathrm{~d} t, \\
\mathscr{I}_{\alpha} f(x) & =\int_{\mathbb{R}^{d}} \mathscr{K}_{\alpha}(x, y) f(y) \mathrm{d} y .
\end{aligned}
$$

The following lemma plays a key role in the proof of our main theorems, which can be found in Proposition 8 of [20] (see also [7], Lemma 3.7).

Lemma 6 (see [20]). Let $V \in R H_{s}$ with $s \geq d / 2$ and $0<\alpha<d$. For every positive integer $N \geq 1$, there exists a positive constant $C_{N}>0$ such that, for all $x$ and $y$ in $\mathbb{R}^{d}$,

$$
\left|\mathscr{K}_{\alpha}(x, y)\right| \leq C_{N}\left(1+\frac{|x-y|}{\rho(x)}\right)^{-N} \frac{1}{|x-y|^{d-\alpha}} .
$$

Proof of Theorem 4. By definition, we only have to show that for any given ball $B=B\left(x_{0}, r\right)$ of $\mathbb{R}^{d}$, there is some $\vartheta>0$ such that

$$
\left(\frac{1}{w^{q}(B)^{K q / p}} \int_{B}\left|\mathscr{I}_{\alpha} f(x)\right|^{q} w^{q}(x) \mathrm{d} x\right)^{1 / q} \leq C \cdot\left(1+\frac{r}{\rho\left(x_{0}\right)}\right)^{9},
$$

holds for any $f \in L_{\rho, \infty}^{p, \kappa}\left(w^{p}, w^{q}\right)$ with $1<p<q<\infty$ and $0<\kappa<p / q$. Suppose that $f \in L_{\rho, \theta}^{p, \kappa}\left(w^{p}, w^{q}\right)$ for some $\theta>0$ and $w \in A_{p, q}^{\rho, \theta^{\prime}}$ for some $\theta^{\prime}>0$. We decompose the function $f$ as

$$
\left\{\begin{array}{l}
f=f_{1}+f_{2} \in L_{\rho, \theta}^{p, \kappa}\left(w^{p}, w^{q}\right) \\
f_{1}=f \cdot \chi_{2 B} ; \\
f_{2}=f \cdot \chi_{(2 B)},
\end{array}\right.
$$

where $2 B$ is the ball centered at $x_{0}$ of radius $2 r>0$ and $\chi_{2 B}$ is the characteristic function of $2 B$. Then, by the linearity of $\mathscr{I}_{\alpha}$, we write

$$
\begin{aligned}
& \left(\frac{1}{w^{q}(B)^{\kappa q / p}} \int_{B}\left|\mathscr{I}_{\alpha} f(x)\right|^{q} w^{q}(x) \mathrm{d} x\right)^{1 / q} \\
& \leq\left(\frac{1}{w^{q}(B)^{\kappa q / p}} \int_{B}\left|\mathscr{I}_{\alpha} f_{1}(x)\right|^{q} w^{q}(x) \mathrm{d} x\right)^{1 / q} \\
& +\left(\frac{1}{w^{q}(B)^{\kappa q / p}} \int_{B}\left|\mathscr{I}_{\alpha} f_{2}(x)\right|^{q} w^{q}(x) \mathrm{d} x\right)^{1 / q} \\
& \quad:=I_{1}+I_{2} .
\end{aligned}
$$
get

$$
\begin{aligned}
I_{1} & =\left(\frac{1}{w^{q}(B)^{\kappa q / p}} \int_{B}\left|\mathscr{I}_{\alpha} f_{1}(x)\right|^{q} w^{q}(x) \mathrm{d} x\right)^{1 / q} \\
& \leq C \cdot \frac{1}{w^{q}(B)^{\kappa / p}}\left(\int_{\mathbb{R}^{d}}\left|f_{1}(x)\right|^{p} w^{p}(x) \mathrm{d} x\right)^{1 / p} \\
& =C \cdot \frac{1}{w^{q}(B)^{\kappa / p}}\left(\int_{2 B}|f(x)|^{p} w^{p}(x) \mathrm{d} x\right)^{1 / p} \\
& \leq C\|f\|_{L_{\rho, \theta}^{p, \kappa}\left(w^{p}, w^{q}\right)} \cdot \frac{w^{q}(2 B)^{\kappa / p}}{w^{q}(B)^{\kappa / p}} \cdot\left(1+\frac{2 r}{\rho\left(x_{0}\right)}\right)^{\theta} .
\end{aligned}
$$

Since $w \in A_{p, q}^{\rho, \theta^{\prime}}$ with $1<p<q<\infty$ and $0<\theta^{\prime}<\infty$, then we know that $w^{q} \in A_{t}^{\rho, \theta^{\prime} \cdot\left(1 /\left((1 / q)+\left(1 / p^{\prime}\right)\right)\right)}$ with $t=1+q / p^{\prime}$ according to Lemma 5 . Now we claim that, for every weight $v \in A_{t}^{\rho, \tau}$ and every ball $B$ in $\mathbb{R}^{d}$, there exists a dimensional constant $C>0$ independent of $v$ and $B$ such that

$$
v\left(2 B\left(x_{0}, r\right)\right) \leq C \cdot\left(1+\frac{2 r}{\rho\left(x_{0}\right)}\right)^{t \tau} v\left(B\left(x_{0}, r\right)\right) .
$$

In fact, for $1<t<\infty$, by Hölder's inequality and the definition of $A_{t}^{\rho, \tau}$, we have

$$
\begin{gathered}
\frac{1}{|2 B|} \int_{2 B}|\hbar(x)| \mathrm{d} x=\frac{1}{|2 B|} \int_{2 B}|\hbar(x)| v(x)^{1 / t} v(x)^{-1 / t} \mathrm{~d} x \\
\quad \leq \frac{1}{|2 B|}\left(\int_{2 B}|\hbar(x)|^{t} v(x) \mathrm{d} x\right)^{1 / t}\left(\int_{2 B} v(x)^{-t^{\prime} / t} \mathrm{~d} x\right)^{1 / t^{\prime}} \\
\quad \leq \frac{C_{v}}{v(2 B)^{1 / t}}\left(\int_{2 B}|\hbar(x)|^{t} v(x) \mathrm{d} x\right)^{1 / t}\left(1+\frac{2 r}{\rho\left(x_{0}\right)}\right)^{\tau} .
\end{gathered}
$$

If we take $\hbar(x)=\chi_{B}(x)$, then the above expression becomes

$$
\frac{|B|}{|2 B|} \leq C_{v} \cdot \frac{v(B)^{1 / t}}{v(2 B)^{1 / t}}\left(1+\frac{2 r}{\rho\left(x_{0}\right)}\right)^{\tau},
$$

which in turn implies (66). Also observe that 


$$
t \theta^{\prime} \cdot \frac{1}{(1 / q)+\left(1 / p^{\prime}\right)}=\left(1+\frac{q}{p^{\prime}}\right) \theta^{\prime} \cdot \frac{1}{(1 / q)+\left(1 / p^{\prime}\right)}=q \theta^{\prime} .
$$

Therefore, in view of (66) and (19),

$$
\begin{aligned}
I_{1} & \leq C_{w}\|f\|_{L_{\rho, \theta}^{p, \kappa}\left(w^{p}, w^{q}\right)} \cdot\left(1+\frac{2 r}{\rho\left(x_{0}\right)}\right)^{\left(q \theta^{\prime}\right) \cdot(\kappa / p)} \cdot\left(1+\frac{2 r}{\rho\left(x_{0}\right)}\right)^{\theta} \\
& =C_{w}\|f\|_{L_{\rho, \theta}^{p, \kappa}\left(w^{p}, w^{q}\right)} \cdot\left(1+\frac{2 r}{\rho\left(x_{0}\right)}\right)^{\vartheta^{\prime}} \leq C \cdot\left(1+\frac{r}{\rho\left(x_{0}\right)}\right)^{\vartheta^{\prime}},
\end{aligned}
$$

where $\vartheta^{\prime}:=\left(q \theta^{\prime} \kappa\right) / p+\theta$. For the other term $I_{2}$, notice that, for any $x \in B$ and $y \in(2 B)^{c}$, one has $|x-y| \approx\left|x_{0}-y\right|$. It then follows from Lemma 6 that, for any $x \in B\left(x_{0}, r\right)$ and any positive integer $N$,

$$
\begin{aligned}
\left|\mathscr{F}_{\alpha} f_{2}(x)\right| \leq & \int_{(2 B)^{c}}\left|\mathscr{K}_{\alpha}(x, y)\right| \cdot|f(y)| \mathrm{d} y \\
\leq & C_{N} \int_{(2 B)^{c}}\left(1+\frac{|x-y|}{\rho(x)}\right)^{-N} \frac{1}{|x-y|^{d-\alpha}} \cdot|f(y)| \mathrm{d} y \\
\leq & C_{N, d} \int_{(2 B)^{c}}\left(1+\frac{\left|x_{0}-y\right|}{\rho(x)}\right)^{-N} \frac{1}{\left|x_{0}-y\right|^{d-\alpha}} \cdot|f(y)| \mathrm{d} y \\
= & C_{N, d} \sum_{k=1}^{\infty} \int_{2^{k+1} B \mid 2^{k} B}\left(1+\frac{\left|x_{0}-y\right|}{\rho(x)}\right)^{-N} \\
& \cdot \frac{1}{\left|x_{0}-y\right|^{d-\alpha} \cdot|f(y)| \mathrm{d} y} \\
\leq & C_{N, d} \sum_{k=1}^{\infty} \frac{1}{\left|2^{k+1} B\right|^{1-(\alpha / d)}} \int_{2^{k+1} B \mid 2^{k} B} \\
& \cdot\left(1+\frac{2^{k} r}{\rho(x)}\right)^{-N}|f(y)| \mathrm{d} y .
\end{aligned}
$$

In view of (4) in Lemma 1 and (19), we further obtain

$$
\begin{aligned}
\left|\mathscr{F}_{\alpha} f_{2}(x)\right| \leq & C \sum_{k=1}^{\infty} \frac{1}{\left|2^{k+1} B\right|^{1-(\alpha / d)}} \int_{2^{k+1} B}\left(1+\frac{r}{\rho\left(x_{0}\right)}\right)^{N \cdot\left(N_{0} /\left(N_{0}+1\right)\right)} \\
& \cdot\left(1+\frac{2^{k} r}{\rho\left(x_{0}\right)}\right)^{-N}|f(y)| \mathrm{d} y \\
\leq & C \sum_{k=1}^{\infty} \frac{1}{\left|2^{k+1} B\right|^{1-(\alpha / d)}} \int_{2^{k+1} B}\left(1+\frac{r}{\rho\left(x_{0}\right)}\right)^{N \cdot\left(N_{0} /\left(N_{0}+1\right)\right)} \\
& \left(1+\frac{2^{k+1} r}{\rho\left(x_{0}\right)}\right)^{-N}|f(y)| \mathrm{d} y .
\end{aligned}
$$

We consider each term in the sum of (72) separately. By using Hölder's inequality and $A_{p, q}^{\rho, \theta^{\prime}}$ condition on $w$, we obtain that, for each $k \geq 1$,

$$
\frac{1}{\left|2^{k+1} B\right|^{1-(\alpha / d)}} \int_{2^{k+1} B}|f(y)| \mathrm{d} y \leq \frac{1}{\left|2^{k+1} B\right|^{1-(\alpha / d)}}
$$

$$
\begin{aligned}
& \cdot\left(\int_{2^{k+1} B}|f(y)|^{p} w^{p}(y) \mathrm{d} y\right)^{1 / p} \\
& \cdot\left(\int_{2^{k+1} B} w(y)^{-p^{\prime}} \mathrm{d} y\right)^{1 / p^{\prime}} \\
& \leq C\|f\|_{L_{,, \theta}^{p, k}\left(w^{p}, w^{q}\right)} \cdot \frac{w^{q}\left(2^{k+1} B\right)^{k / p}}{w^{q}\left(2^{k+1} B\right)^{1 / q}} \\
& \cdot\left(1+\frac{2^{k+1} r}{\rho\left(x_{0}\right)}\right)^{\theta}\left(1+\frac{2^{k+1} r}{\rho\left(x_{0}\right)}\right)^{\theta^{\prime}} .
\end{aligned}
$$

Hence,

$$
\begin{aligned}
I_{2} \leq & C\|f\|_{L_{\rho, \theta}^{p, \kappa}\left(w^{p}, w^{q}\right)} \cdot \frac{w^{q}(B)^{1 / q}}{w^{q}(B)^{\kappa / p}} \sum_{k=1}^{\infty} \frac{w^{q}\left(2^{k+1} B\right)^{\kappa / p}}{w^{q}\left(2^{k+1} B\right)^{1 / q}} \\
& \cdot\left(1+\frac{r}{\rho\left(x_{0}\right)}\right)^{N \cdot\left(N_{0} / N_{0}+1\right)}\left(1+\frac{2^{k+1} r}{\rho\left(x_{0}\right)}\right)^{-N+\theta+\theta^{\prime}} \\
= & C\|f\|_{L_{\rho, \theta}^{p, \kappa}\left(w^{p}, w^{q}\right)}\left(1+\frac{r}{\rho\left(x_{0}\right)}\right)^{N \cdot\left(N_{0} / N_{0}+1\right)} \\
& \cdot \sum_{k=1}^{\infty} \frac{w^{q}(B)^{1 / q-\kappa / p}}{w^{q}\left(2^{k+1} B\right)^{1 / q-\kappa / p}}\left(1+\frac{2^{k+1} r}{\rho\left(x_{0}\right)}\right)^{-N+\theta+\theta^{\prime}} .
\end{aligned}
$$

Recall that $w^{q} \in A_{t}^{\rho, \theta^{\prime} \cdot\left(1 /(1 / q)+\left(1 / p^{\prime}\right)\right)}$ with $t=1+q / p^{\prime}$ and $0<\theta^{\prime}<\infty$, then there exist two positive numbers $\delta, \eta>0$ such that (25) holds. This allows us to obtain

$$
\begin{aligned}
I_{2} \leq & C\|f\|_{L_{\rho, \theta}^{p, \kappa}\left(w^{p}, w^{q)}\right.}\left(1+\frac{r}{\rho\left(x_{0}\right)}\right)^{N \cdot\left(N_{0} /\left(N_{0}+1\right)\right)} \\
& \cdot \sum_{k=1}^{\infty}\left(\frac{|B|}{\left|2^{k+1} B\right|}\right)^{\delta((1 / q)-(\kappa / p))} \times\left(1+\frac{2^{k+1} r}{\rho\left(x_{0}\right)}\right)^{\eta((1 / q)-(\kappa / p))} \\
& \cdot\left(1+\frac{2^{k+1} r}{\rho\left(x_{0}\right)}\right)^{-N+\theta+\theta^{\prime}} \\
= & C\|f\|_{L_{\rho, \theta}^{p, k}\left(w^{p}, w^{q}\right)}\left(1+\frac{r}{\rho\left(x_{0}\right)}\right)^{N \cdot\left(N_{0} /\left(N_{0}+1\right)\right)} \\
& \times \sum_{k=1}^{\infty}\left(\frac{|B|}{\left|2^{k+1} B\right|}\right)^{\delta((1 / q)-(\kappa / p))}\left(1+\frac{2^{k+1} r}{\rho\left(x_{0}\right)}\right)^{-N+\theta+\theta^{\prime}+\eta((1 / q)-(\kappa / p))} .
\end{aligned}
$$

Thus, by choosing $N$ large enough so that $N>\theta+\theta^{\prime}+\eta((1 / q)-(\kappa / p))$, and the last series is convergent, we then have 


$$
\begin{aligned}
I_{2} \leq & C\|f\|_{L_{\rho, \theta}^{p, \kappa}\left(w^{p}, w^{q}\right)}\left(1+\frac{r}{\rho\left(x_{0}\right)}\right)^{N \cdot\left(N_{0} /\left(N_{0}+1\right)\right)} \\
& \cdot \sum_{k=1}^{\infty}\left(\frac{|B|}{\left|2^{k+1} B\right|}\right)^{\delta((1 / q)-(\kappa / p))} \\
\leq & C\left(1+\frac{r}{\rho\left(x_{0}\right)}\right)^{N \cdot\left(N_{0} /\left(N_{0}+1\right)\right)}
\end{aligned}
$$

where the last inequality follows from the fact that $(1 / q)-(\kappa / p)>0$. Summing up the above estimates for $I_{1}$ and $I_{2}$ and letting $\vartheta=\max \left\{\vartheta^{\prime}, N \cdot\left(N_{0} /\left(N_{0}+1\right)\right)\right\}$, we obtain our desired inequality (62). This completes the proof of Theorem 4.

Proof of Theorem 5. To prove Theorem 5, by definition, it suffices to prove that, for each given ball $B=B\left(x_{0}, r\right)$ of $\mathbb{R}^{d}$, there is some $\vartheta>0$ such that

$$
\frac{1}{w^{q}(B)^{\kappa}} \sup _{\lambda>0} \lambda \cdot\left[w^{q}\left(\left\{x \in B:\left|\mathscr{I}_{\alpha} f(x)\right|>\lambda\right\}\right)\right]^{1 / q} \leq C \cdot\left(1+\frac{r}{\rho\left(x_{0}\right)}\right)^{9},
$$

holds for any $f \in L_{\rho, \infty}^{1, \kappa}\left(w, w^{q}\right)$ with $1<q<\infty$ and $0<\kappa<1 / q$. Now suppose that $f \in L_{\rho, \theta}^{1, \kappa}\left(w, w^{q}\right)$ for some $\theta>0$ and $w \in A_{1, q}^{\rho, \theta^{\prime}}$ for some $\theta^{\prime}>0$. We decompose the function $f$ as

$$
\left\{\begin{array}{l}
f=f_{1}+f_{2} \in L_{\rho, \theta}^{1, \kappa}\left(w, w^{q}\right), \\
f_{1}=f \cdot \chi_{2 B}, \\
f_{2}=f \cdot \chi_{(2 B)^{c}} .
\end{array}\right.
$$

Then, for any given $\lambda>0$, by the linearity of $\mathscr{I}_{\alpha}$, we can write

$$
\begin{aligned}
& \frac{1}{w^{q}(B)^{\kappa}} \lambda \cdot\left[w^{q}\left(\left\{x \in B:\left|\mathscr{I}_{\alpha} f(x)\right|>\lambda\right\}\right)\right]^{1 / q} \leq \frac{1}{w^{q}(B)^{\kappa}} \lambda \\
& \cdot\left[w^{q}\left(\left\{x \in B:\left|\mathscr{I}_{\alpha} f_{1}(x)\right|>\frac{\lambda}{2}\right\}\right)\right]^{1 / q} \\
& +\frac{1}{w^{q}(B)^{\kappa}} \lambda \cdot\left[w^{q}\left(\left\{x \in B:\left|\mathscr{I}_{\alpha} f_{2}(x)\right|>\frac{\lambda}{2}\right\}\right)\right]^{1 / q} \\
& :=I_{1}^{\prime}+I_{2}^{\prime} .
\end{aligned}
$$

We first give the estimate for the term $I_{1}^{\prime}$. By Theorem 2, we get

$$
\begin{aligned}
I_{1}^{\prime} & =\frac{1}{w^{q}(B)^{\kappa}} \lambda \cdot\left[w^{q}\left(\left\{x \in B:\left|\mathscr{I}_{\alpha} f_{1}(x)\right|>\frac{\lambda}{2}\right\}\right)\right]^{1 / q} \\
& \leq C \cdot \frac{1}{w^{q}(B)^{\kappa}}\left(\int_{\mathbb{R}^{d}}\left|f_{1}(x)\right| w(x) \mathrm{d} x\right) \\
& =C \cdot \frac{1}{w^{q}(B)^{\kappa}}\left(\int_{2 B}|f(x)| w(x) \mathrm{d} x\right) \\
& \leq C\|f\|_{L_{\rho, \theta}^{1, \kappa}\left(w, w^{q}\right)} \cdot \frac{w^{q}(2 B)^{\kappa}}{w^{q}(B)^{\kappa}} \cdot\left(1+\frac{2 r}{\rho\left(x_{0}\right)}\right)^{\theta} .
\end{aligned}
$$

Since $w \in A_{1, q}^{\rho, \theta^{\prime}}$ with $1<q<\infty$ and $0<\theta^{\prime}<\infty$, then we know that $w^{q} \in A_{1}^{\rho, \theta^{\prime} \cdot q}$ according to Lemma 5. We now claim that there exists a dimensional constant $C>0$ independent of $v$ and $B$ such that, for every weight $v \in A_{1}^{\rho, \tau}$ and every ball $B$ in $\mathbb{R}^{d}$,

$$
v\left(2 B\left(x_{0}, r\right)\right) \leq C \cdot\left(1+\frac{2 r}{\rho\left(x_{0}\right)}\right)^{\tau} v\left(B\left(x_{0}, r\right)\right) .
$$

Similar to the proof of (66), by the definition of $A_{1}^{\rho, \tau}$, we can deduce that

$$
\begin{aligned}
\frac{1}{|2 B|} \int_{2 B}|\hbar(x)| \mathrm{d} x \leq & \frac{C_{v}}{v(2 B)} \cdot \underset{x \in 2 B}{\operatorname{essinf}} v(x) \\
& \cdot\left(\int_{2 B}|\hbar(x)| \mathrm{d} x\right)\left(1+\frac{2 r}{\rho\left(x_{0}\right)}\right)^{\tau} \\
\leq & \frac{C_{v}}{v(2 B)}\left(\int_{2 B}|\hbar(x)| v(x) \mathrm{d} x\right)\left(1+\frac{2 r}{\rho\left(x_{0}\right)}\right)^{\tau} .
\end{aligned}
$$

If we choose $\hbar(x)=\chi_{B}(x)$, then the above expression becomes

$$
\frac{|B|}{|2 B|} \leq C_{v} \cdot \frac{v(B)}{v(2 B)}\left(1+\frac{2 r}{\rho\left(x_{0}\right)}\right)^{\tau}
$$

which in turn implies (81). Therefore, in view of (81) and (19),

$$
\begin{aligned}
& I_{1}^{\prime} \leq C \cdot\left(1+\frac{2 r}{\rho\left(x_{0}\right)}\right)^{\left(\theta^{\prime} q\right) \cdot \kappa} \cdot\left(1+\frac{2 r}{\rho\left(x_{0}\right)}\right)^{\theta} \\
& =C \cdot\left(1+\frac{2 r}{\rho\left(x_{0}\right)}\right)^{\vartheta^{\prime}} \leq C \cdot\left(1+\frac{r}{\rho\left(x_{0}\right)}\right)^{\vartheta^{\prime}},
\end{aligned}
$$

where $\vartheta^{\prime}:=\theta^{\prime} q \kappa+\theta$. As for the second term $I_{2}^{\prime}$, by using the pointwise inequality (72) and Chebyshev's inequality, we deduce that 


$$
\begin{aligned}
I_{2}^{\prime}= & \frac{1}{w^{q}(B)^{\kappa}} \lambda \cdot\left[w^{q}\left(\left\{x \in B:\left|\mathscr{I}_{\alpha} f_{2}(x)\right|>\frac{\lambda}{2}\right\}\right)\right]^{1 / q} \\
\leq & \frac{2}{w^{q}(B)^{\kappa}}\left(\int_{B}\left|\mathscr{I}_{\alpha} f_{2}(x)\right|^{q} w^{q}(x) \mathrm{d} x\right)^{1 / q} \\
\leq & C \cdot \frac{w^{q}(B)^{1 / q}}{w^{q}(B)^{\kappa}} \sum_{k=1}^{\infty} \frac{1}{\left|2^{k+1} B\right|^{1-(\alpha / d)}} \int_{2^{k+1} B} \\
& \cdot\left(1+\frac{r}{\rho\left(x_{0}\right)}\right)^{N \cdot\left(N_{0} /\left(N_{0}+1\right)\right)}\left(1+\frac{2^{k+1} r}{\rho\left(x_{0}\right)}\right)^{-N}|f(y)| \mathrm{d} y .
\end{aligned}
$$

We consider each term in the sum of (85) separately. By Lemma 4, we know that $w \in A_{1}^{\rho, \theta^{\prime}} \cap \mathrm{RH}_{q}^{\rho, \theta^{\prime}}$ because $w^{q}$ is in $A_{1}^{\rho, \theta^{\prime} \cdot q}$. Thus, for each $k \geq 1$, we compute

$$
\begin{aligned}
& \frac{1}{\left|2^{k+1} B\right|^{1-(\alpha / d)}} \int_{2^{k+1} B}|f(y)| \mathrm{d} y \leq C_{w} \cdot \frac{\left|2^{k+1} B\right|^{\alpha / d}}{w\left(2^{k+1} B\right)} \\
& \cdot \underset{y \in 2^{k+1} B}{\operatorname{essinf}} w(y)\left(\int_{2^{k+1} B}|f(y)| \mathrm{d} y\right)\left(1+\frac{2^{k+1} r}{\rho\left(x_{0}\right)}\right)^{\theta^{\prime}} \\
& \leq C_{w} \cdot \frac{\left|2^{k+1} B\right|^{\alpha / d}}{w\left(2^{k+1} B\right)}\left(\int_{2^{k+1} B}|f(y)| w(y) \mathrm{d} y\right)\left(1+\frac{2^{k+1} r}{\rho\left(x_{0}\right)}\right)^{\theta^{\prime}} \\
& \leq C\|f\|_{L_{\rho, \theta}^{1, k}\left(w, w^{q}\right)} \cdot \frac{\left|2^{k+1} B\right|^{\alpha / d}}{w\left(2^{k+1} B\right)} w^{q}\left(2^{k+1} B\right)^{\kappa} \\
& .\left(1+\frac{2^{k+1} r}{\rho\left(x_{0}\right)}\right)^{\theta}\left(1+\frac{2^{k+1} r}{\rho\left(x_{0}\right)}\right)^{\theta^{\prime}} .
\end{aligned}
$$

In addition, by reverse Hölder-type inequality $\left(w \in R H_{q}^{\rho, \theta^{\prime}}\right)$, we can see that

$$
\begin{aligned}
w^{q}\left(2^{k+1} B\right)^{1 / q} & =\left(\int_{2^{k+1} B} w^{q}(y) \mathrm{d} y\right)^{1 / q} \\
& \leq C\left|2^{k+1} B\right|^{1 / q}\left(\frac{1}{\left|2^{k+1} B\right|} \int_{2^{k+1} B} w(y) \mathrm{d} y\right)\left(1+\frac{2^{k+1} r}{\rho\left(x_{0}\right)}\right)^{\theta^{\prime}} .
\end{aligned}
$$

This indicates that

$$
\frac{\left|2^{k+1} B\right|^{\alpha / d}}{w\left(2^{k+1} B\right)} \leq C \cdot \frac{1}{w^{q}\left(2^{k+1} B\right)^{1 / q}}\left(1+\frac{2^{k+1} r}{\rho\left(x_{0}\right)}\right)^{\theta^{\prime}} .
$$

Consequently,

$$
\begin{gathered}
I_{2}^{\prime \leq} C\|f\|_{L_{\rho, \theta}^{1, \kappa}\left(w, w^{q}\right)} \cdot \frac{w^{q}(B)^{1 / q}}{w^{q}(B)^{\kappa}} \sum_{k=1}^{\infty} \frac{w^{q}\left(2^{k+1} B\right)^{\kappa}}{w^{q}\left(2^{k+1} B\right)^{1 / q}} \\
\cdot\left(1+\frac{r}{\rho\left(x_{0}\right)}\right)^{N \cdot\left(N_{0} /\left(N_{0}+1\right)\right)}\left(1+\frac{2^{k+1} r}{\rho\left(x_{0}\right)}\right)^{-N+\theta+2 \theta^{\prime}} \\
=C\|f\|_{L_{\rho, \theta}^{1, \kappa}\left(w, w^{q}\right)}\left(1+\frac{r}{\rho\left(x_{0}\right)}\right)^{N \cdot\left(N_{0} /\left(N_{0}+1\right)\right)} \\
\cdot \sum_{k=1}^{\infty} \frac{w^{q}(B)^{1 / q-\kappa}}{w^{q}\left(2^{k+1} B\right)^{1 / q-\kappa}}\left(1+\frac{2^{k+1} r}{\rho\left(x_{0}\right)}\right)^{-N+\theta+2 \theta^{\prime}}
\end{gathered}
$$

Recall that $w^{q} \in A_{1}^{\rho, \theta^{\prime} \cdot q}$ with $0<\theta^{\prime}<\infty$ and $1<q<\infty$, then there exist two positive numbers $\delta^{\prime}, \eta^{\prime}>0$ such that (25) holds. Therefore,

$$
\begin{aligned}
I_{2}^{\prime} \leq & C\|f\|_{L_{\rho, \theta}^{1, \kappa}\left(w, w^{q}\right)}\left(1+\frac{r}{\rho\left(x_{0}\right)}\right)^{N \cdot\left(N_{0} /\left(N_{0}+1\right)\right)} \sum_{k=1}^{\infty}\left(\frac{|B|}{\left|2^{k+1} B\right|}\right)^{\delta^{\prime}(1 / q-\kappa)} \\
& \times\left(1+\frac{2^{k+1} r}{\rho\left(x_{0}\right)}\right)^{\eta^{\prime}(1 / q-\kappa)}\left(1+\frac{2^{k+1} r}{\rho\left(x_{0}\right)}\right)^{-N+\theta+2 \theta^{\prime}} \\
= & C\|f\|_{L_{\rho, \theta}^{1, \kappa}\left(w, w^{q}\right)}\left(1+\frac{r}{\rho\left(x_{0}\right)}\right)^{N \cdot\left(N_{0} /\left(N_{0}+1\right)\right)} \\
& \times \sum_{k=1}^{\infty}\left(\frac{|B|}{\left|2^{k+1} B\right|}\right)^{\delta^{\prime}(1 / q-\kappa)}\left(1+\frac{2^{k+1} r}{\rho\left(x_{0}\right)}\right)^{-N+\theta+2 \theta^{\prime}+\eta^{\prime}(1 / q-\kappa)}
\end{aligned}
$$

By selecting $N$ large enough so that $N>\theta+2 \theta^{\prime}+\eta^{\prime}(1 / q-\kappa)$, we thus have

$$
\begin{aligned}
I_{2}^{\prime} & \leq C\left(1+\frac{r}{\rho\left(x_{0}\right)}\right)^{N \cdot\left(N_{0} /\left(N_{0}+1\right)\right)} \sum_{k=1}^{\infty}\left(\frac{|B|}{\left|2^{k+1} B\right|}\right)^{\delta^{\prime}(1 / q-\kappa)} \\
& \leq C\left(1+\frac{r}{\rho\left(x_{0}\right)}\right)^{N \cdot\left(N_{0} /\left(N_{0}+1\right)\right)}
\end{aligned}
$$

where the last inequality is due to $0<\kappa<1 / q$. Let $\vartheta=\max \left\{\vartheta^{\prime}, N \cdot\left(N_{0} /\left(N_{0}+1\right)\right)\right\}$. Here $N$ is an appropriate constant. Summing up the above estimates for $I_{1}^{\prime}$ and $I_{2}^{\prime}$, and then taking the supremum over all $\lambda>0$, we obtain the desired inequality (77). This finishes the proof of Theorem 5 .

\section{Proof of Theorem 6}

For the result involving commutators of fractional integrals associated with Schrödinger operators, we need the following properties of $\mathrm{BMO}_{\rho, \infty}\left(\mathbb{R}^{d}\right)$ functions, which are extensions of well-known properties of $\mathrm{BMO}\left(\mathbb{R}^{d}\right)$ functions. 
Lemma 7. If $b \in B M O_{\rho, \infty}\left(\mathbb{R}^{d}\right)$ and $w \in A_{p}^{\rho, \infty}$ with $1 \leq p<\infty$, then there exist positive constants $C>0$ and $\mu>0$ such that for every ball $B=B\left(x_{0}, r\right)$ in $\mathbb{R}^{d}$, we have

$$
\left(\int_{B}\left|b(x)-b_{B}\right|^{p} w(x) \mathrm{d} x\right)^{1 / p} \leq C \cdot w(B)^{1 / p}\left(1+\frac{r}{\rho\left(x_{0}\right)}\right)^{\mu},
$$

where $b_{B}=(1 /|B|) \int_{B} b(y) d y$.

Proof. We may assume that $b \in \mathrm{BMO}_{\rho, \theta}\left(\mathbb{R}^{d}\right)$ with $0<\theta<\infty$. According to Proposition 3, we can deduce that

$$
\begin{aligned}
\left(\int_{B}\left|b(x)-b_{B}\right|^{p} w(x) \mathrm{d} x\right)^{1 / p} & =\left(\int_{0}^{\infty} p \lambda^{p-1} w\left(\left\{x \in B:\left|b(x)-b_{B}\right|>\lambda\right\}\right) \mathrm{d} \lambda\right)^{1 / p} \\
& \leq C_{1}^{1 / p} \cdot w(B)^{1 / p}\left\{\int_{0}^{\infty} p \lambda^{p-1} \exp \left[-\left(1+\frac{r}{\rho\left(x_{0}\right)}\right)^{-\theta^{*}} \frac{C_{2} \lambda}{\|b\|_{\mathrm{BMO}_{\rho, \theta}}}\right]\left(1+\frac{r}{\rho\left(x_{0}\right)}\right)^{\eta} \mathrm{d} \lambda\right\}^{1 / p} \\
& =C_{1}^{1 / p} \cdot w(B)^{1 / p}\left\{\int_{0}^{\infty} p \lambda^{p-1} \exp \left[-\left(1+\frac{r}{\rho\left(x_{0}\right)}\right)^{-\theta^{*}} \frac{C_{2} \lambda}{\|b\|_{\mathrm{BMO}_{\rho, \theta}}}\right] \mathrm{d} \lambda\right\}^{1 / p}\left(1+\frac{r}{\rho\left(x_{0}\right)}\right)^{\eta / p} .
\end{aligned}
$$

By a change of variables, we thus have

$$
\begin{aligned}
& \left(\int_{B}\left|b(x)-b_{B}\right|^{p} w(x) \mathrm{d} x\right)^{1 / p} \leq C_{1}^{1 / p} \cdot w(B)^{1 / p}\left(\int_{0}^{\infty} p s^{p-1} e^{-s} \mathrm{~d} s\right)^{1 / p}\left(\frac{\|b\|_{\mathrm{BMO}_{\rho, \theta}}}{C_{2}}\right)\left(1+\frac{r}{\rho\left(x_{0}\right)}\right)^{\theta^{*}}\left(1+\frac{r}{\rho\left(x_{0}\right)}\right)^{\eta / p} \\
& =\left[C_{1} p \Gamma(p)\right]^{1 / p}\left(\frac{\|b\|_{\mathrm{BMO}_{\rho, \theta}}}{C_{2}}\right) \cdot w(B)^{1 / p}\left(1+\frac{r}{\rho\left(x_{0}\right)}\right)^{\theta^{*}+\eta / p},
\end{aligned}
$$

which yields the desired inequality (92), if we choose $C=$ $\left[C_{1} p \Gamma(p)\right]^{1 / p} C_{2}^{-1}\|b\|_{\mathrm{BMO}_{\rho, \theta}}$ and $\mu=\theta^{*}+\eta / p$.

Lemma 8. If $b \in B M O_{\rho, \theta}\left(\mathbb{R}^{d}\right)$ with $0<\theta<\infty$, then for any positive integer $k$, there exists a positive constant $C>0$ such that, for every ball $B=B\left(x_{0}, r\right)$ in $\mathbb{R}^{d}$, we have

$$
\left|b_{2^{k+1} B}-b_{B}\right| \leq C \cdot(k+1)\left(1+\frac{2^{k+1} r}{\rho\left(x_{0}\right)}\right)^{\theta} .
$$

Proof. For any positive integer $k$, we have

$$
\begin{aligned}
\left|b_{2^{k+1} B}-b_{B}\right| & \leq \sum_{j=1}^{k+1}\left|b_{2^{j} B}-b_{2^{j-1} B}\right| \\
& =\sum_{j=1}^{k+1}\left|\frac{1}{\left|2^{j-1} B\right|} \int_{2^{j-1} B}\left[b_{2^{j} B}-b(y)\right] \mathrm{d} y\right| \\
& \leq \sum_{j=1}^{k+1} \frac{2^{d}}{\left|2^{j} B\right|} \int_{2^{j} B}\left|b(y)-b_{2^{j} B}\right| \mathrm{d} y \\
& \leq C_{b, d}\|b\|_{\mathrm{BMO}_{\rho, \theta}} \sum_{j=1}^{k+1}\left(1+\frac{2^{j} r}{\rho\left(x_{0}\right)}\right)^{\theta} .
\end{aligned}
$$

Since for any $1 \leq j \leq k+1$, the following estimate

$$
\left(1+\frac{2^{j} r}{\rho\left(x_{0}\right)}\right)^{\theta} \leq\left(1+\frac{2^{k+1} r}{\rho\left(x_{0}\right)}\right)^{\theta}
$$

holds trivially and hence

$$
\left|b_{2^{k+1} B}-b_{B}\right| \leq C \sum_{j=1}^{k+1}\left(1+\frac{2^{k+1} r}{\rho\left(x_{0}\right)}\right)^{\theta}=C \cdot(k+1)\left(1+\frac{2^{k+1} r}{\rho\left(x_{0}\right)}\right)^{\theta} .
$$

This is just our desired conclusion.

Now, we are ready to prove the main result of this section.

Proof of Theorem 6. By definition, we only need to prove that, for an arbitrary ball $B=B\left(x_{0}, r\right)$ of $\mathbb{R}^{d}$ and $0<\alpha<d$, there is some $\vartheta>0$ such that

$$
\left(\frac{1}{w^{q}(B)^{K q / p}} \int_{B}\left|\left[b, \mathscr{I}_{\alpha}\right] f(x)\right|^{q} w^{q}(x) \mathrm{d} x\right)^{1 / q} \leq C \cdot\left(1+\frac{r}{\rho\left(x_{0}\right)}\right)^{9},
$$

holds for any $f \in L_{\rho, \infty}^{p, \kappa}\left(w^{p}, w^{q}\right)$ with $1<p<q<\infty$ and $0<\kappa<p / q$, whenever $b$ belongs to $\mathrm{BMO}_{\rho, \infty}\left(\mathbb{R}^{d}\right)$. Suppose that $f \in L_{\rho, \theta}^{p, \kappa}\left(w^{p}, w^{q}\right)$ for some $\theta>0, w \in A_{p, q}^{\rho, \theta^{\prime}}$ for some $\theta^{\prime}>0$ as well as $b \in \mathrm{BMO}_{\rho, \theta^{\prime \prime}}\left(\mathbb{R}^{d}\right)$ for some $\theta^{\prime \prime}>0$. As before, we decompose the function $f$ as 


$$
\left\{\begin{array}{l}
f=f_{1}+f_{2} \in L_{\rho, \theta}^{p, \kappa}\left(w^{p}, w^{q}\right) \\
f_{1}=f \cdot \chi_{2 B} \\
f_{2}=f \cdot \chi_{(2 B)^{c}} .
\end{array}\right.
$$

Then by the linearity of $\left[b, \mathscr{I}_{\alpha}\right]$, we write

$$
\begin{aligned}
& \left(\frac{1}{w^{q}(B)^{\kappa q / p}} \int_{B}\left|\left[b, \mathscr{I}_{\alpha}\right] f(x)\right|^{q} w^{q}(x) \mathrm{d} x\right)^{1 / q} \\
& \leq\left(\frac{1}{w^{q}(B)^{\kappa q / p}} \int_{B}\left|\left[b, \mathscr{I}_{\alpha}\right] f_{1}(x)\right|^{q} w^{q}(x) \mathrm{d} x\right)^{1 / q} \\
& \quad+\left(\frac{1}{w^{q}(B)^{\kappa q / p}} \int_{B}\left|\left[b, \mathscr{I}_{\alpha}\right] f_{2}(x)\right|^{q} w^{q}(x) \mathrm{d} x\right)^{1 / q} \\
& :=J_{1}+J_{2} .
\end{aligned}
$$

Now, we give the estimates for $J_{1}$ and $J_{2}$, respectively. According to Theorem 3, we have

$$
\begin{aligned}
J_{1} & \leq C \cdot \frac{1}{w^{q}(B)^{\kappa / p}}\left(\int_{\mathbb{R}^{d}}\left|f_{1}(x)\right|^{p} w^{p}(x) \mathrm{d} x\right)^{1 / p} \\
& =C \cdot \frac{1}{w^{q}(B)^{\kappa / p}}\left(\int_{2 B}|f(x)|^{p} w^{p}(x) \mathrm{d} x\right)^{1 / p} \\
& \leq C\|f\|_{L_{\rho, \theta}^{p, \kappa}\left(w^{p}, w^{q}\right)} \cdot \frac{w^{q}(2 B)^{\kappa / p}}{w^{q}(B)^{\kappa / p}} \cdot\left(1+\frac{2 r}{\rho\left(x_{0}\right)}\right)^{\theta} .
\end{aligned}
$$

Moreover, notice that $w^{q} \in A_{t}^{\rho, \theta^{\prime} \cdot\left(1 /\left((1 / q)+\left(1 / p^{\prime}\right)\right)\right)}$ with $t=$ $1+q / p^{\prime}$ by Lemma 5 , and that

$$
t \theta^{\prime} \cdot \frac{1}{(1 / q)+\left(1 / p^{\prime}\right)}=\left(1+\frac{q}{p^{\prime}}\right) \theta^{\prime} \cdot \frac{1}{(1 / q)+\left(1 / p^{\prime}\right)}=q \theta^{\prime}
$$

Thus, in view of the inequalities (66) and (19), we get

$$
\begin{aligned}
J_{1} & \leq C\|f\|_{L_{\rho, \theta}^{p, \kappa}\left(w^{p}, w^{q}\right)} \cdot\left(1+\frac{2 r}{\rho\left(x_{0}\right)}\right)^{\left(q \theta^{\prime}\right) \cdot(\kappa / p)} \cdot\left(1+\frac{2 r}{\rho\left(x_{0}\right)}\right)^{\theta} \\
& =C\|f\|_{L_{\rho, \theta}^{p, \kappa}\left(w^{p}, w^{q}\right)}\left(1+\frac{2 r}{\rho\left(x_{0}\right)}\right)^{\vartheta^{\prime}} \leq C \cdot\left(1+\frac{r}{\rho\left(x_{0}\right)}\right)^{\vartheta^{\prime}},
\end{aligned}
$$

where $\vartheta^{\prime}:=\left(q \theta^{\prime} \kappa\right) / p+\theta$. On the other hand, by definition (40), we can see that, for any $x \in B\left(x_{0}, r\right)$,

$$
\left|\left[b, \mathscr{I}_{\alpha}\right] f_{2}(x)\right| \leq \int_{\mathbb{R}^{d}}|b(x)-b(y)|\left|\mathscr{K}_{\alpha}(x, y) f_{2}(y)\right| \mathrm{d} y .
$$

Adding and subtracting $b_{B}$ inside the integral, we write

$$
\begin{aligned}
\left|\left[b, \mathscr{I}_{\alpha}\right] f_{2}(x)\right| \leq & \left|b(x)-b_{B}\right| \int_{\mathbb{R}^{d}}\left|\mathscr{K}_{\alpha}(x, y) f_{2}(y)\right| \mathrm{d} y \\
& +\int_{\mathbb{R}^{d}}\left|b(y)-b_{B}\right|\left|\mathscr{K}_{\alpha}(x, y) f_{2}(y)\right| \mathrm{d} y \\
:= & \xi(x)+\zeta(x) .
\end{aligned}
$$

So, we can divide $J_{2}$ into two parts:

$$
\begin{aligned}
J_{2} \leq & \left(\frac{1}{w^{q}(B)^{\kappa q / p}} \int_{B}|\xi(x)|^{q} w^{q}(x) \mathrm{d} x\right)^{1 / q} \\
& +\left(\frac{1}{w^{q}(B)^{\kappa q / p}} \int_{B}|\zeta(x)|^{q} w^{q}(x) \mathrm{d} x\right)^{1 / q} \\
= & J_{3}+J_{4} .
\end{aligned}
$$

To deal with the term $J_{3}$, since $t=1+q / p^{\prime}<q$, one has $w^{q} \in A_{t}^{\rho, \infty} \subset A_{q}^{\rho, \infty}$. From the pointwise estimate (72) and (92) in Lemma 7 , it then follows that

$$
\begin{aligned}
J_{3} \leq & C \cdot \frac{1}{w^{q}(B)^{\kappa / p}}\left(\int_{B}\left|b(x)-b_{B}\right|^{q} w^{q}(x) \mathrm{d} x\right)^{1 / q} \\
& \times \sum_{k=1}^{\infty} \frac{1}{\left|2^{k+1} B\right|^{1-(\alpha / d)}} \int_{2^{k+1} B}\left(1+\frac{r}{\rho\left(x_{0}\right)}\right)^{N \cdot\left(N_{0} /\left(N_{0}+1\right)\right)} \\
& \cdot\left(1+\frac{2^{k+1} r}{\rho\left(x_{0}\right)}\right)^{-N}|f(y)| \mathrm{d} y \\
\leq & C_{b} \cdot \frac{w^{q}(B)^{1 / q}}{w^{q}(B)^{\kappa / p}}\left(1+\frac{r}{\rho\left(x_{0}\right)}\right)^{\mu} \\
& \times \sum_{k=1}^{\infty} \frac{1}{\left|2^{k+1} B\right|^{1-(\alpha / d)}} \int_{2^{k+1} B}\left(1+\frac{r}{\rho\left(x_{0}\right)}\right)^{N \cdot\left(N_{0} /\left(N_{0}+1\right)\right)} \\
& \cdot\left(1+\frac{2^{k+1} r}{\rho\left(x_{0}\right)}\right)^{-N}|f(y)| \mathrm{d} y .
\end{aligned}
$$

Following along the same lines as that of Theorem 4, we are able to show that

$$
\begin{aligned}
J_{3} \leq & C\|f\|_{L_{\rho, \theta}^{p, \kappa}\left(w^{p}, w^{q}\right)}\left(1+\frac{r}{\rho\left(x_{0}\right)}\right)^{\mu}\left(1+\frac{r}{\rho\left(x_{0}\right)}\right)^{N \cdot\left(N_{0} /\left(N_{0}+1\right)\right)} \\
& \times \sum_{k=1}^{\infty} \frac{w^{q}(B)^{(1 / q)-(\kappa / p)}}{w^{q}\left(2^{k+1} B\right)^{(1 / q)-(\kappa / p)}}\left(1+\frac{2^{k+1} r}{\rho\left(x_{0}\right)}\right)^{-N+\theta+\theta^{\prime}} \\
\leq & C\|f\|_{L_{\rho, \theta}^{p, \kappa}\left(w^{p}, w^{q}\right)}\left(1+\frac{r}{\rho\left(x_{0}\right)}\right)^{\mu+N \cdot\left(N_{0} /\left(N_{0}+1\right)\right)} \\
& \times \sum_{k=1}^{\infty}\left(\frac{|B|}{\left|2^{k+1} B\right|}\right)^{\delta((1 / q)-(\kappa / p))}\left(1+\frac{2^{k+1} r}{\rho\left(x_{0}\right)}\right)^{-N+\theta+\theta^{\prime}+\eta((1 / q)-(\kappa / p))} .
\end{aligned}
$$

The last inequality is obtained by using (25). Next, we estimate $\zeta(x)$ for any $x \in B\left(x_{0}, r\right)$. For any positive integer $N$, similar to the proof of (71) and (72), we can also deduce that 


$$
\begin{aligned}
\zeta(x) & =\int_{(2 B)^{c}}\left|b(y)-b_{B}\right|\left|\mathscr{K}_{\alpha}(x, y) f(y)\right| \mathrm{d} y \\
& \leq C_{N} \int_{(2 B)^{c}}\left|b(y)-b_{B}\right|\left(1+\frac{|x-y|}{\rho(x)}\right)^{-N} \frac{1}{|x-y|^{d-\alpha}} \cdot|f(y)| \mathrm{d} y \\
& \leq C_{N, d} \sum_{k=1}^{\infty} \int_{2^{k+1} B \mid 2^{k} B}\left|b(y)-b_{B}\right|\left(1+\frac{\left|x_{0}-y\right|}{\rho(x)}\right)^{-N} \frac{1}{\left|x_{0}-y\right|^{d-\alpha}} \cdot|f(y)| \mathrm{d} y \\
& \leq C_{N, d} \sum_{k=1}^{\infty} \frac{1}{\left.2^{k+1} B\right|^{1-(\alpha / d)}} \int_{2^{k+1} B \mid 2^{k} B}\left|b(y)-b_{B}\right|\left(1+\frac{2^{k} r}{\rho(x)}\right)^{-N}|f(y)| \mathrm{d} y \\
& \leq C \sum_{k=1}^{\infty} \frac{1}{\left|2^{k+1} B\right|^{1-(\alpha / d)}} \int_{2^{k+1} B}\left|b(y)-b_{B}\right|\left(1+\frac{r}{\rho\left(x_{0}\right)}\right)^{N \cdot\left(N_{0} /\left(N_{0}+1\right)\right)}\left(1+\frac{2^{k+1} r}{\rho\left(x_{0}\right)}\right)^{-N}|f(y)| \mathrm{d} y,
\end{aligned}
$$

where in the last inequality we have used (4) in Lemma 1. Hence, by the above pointwise estimate for $\zeta(x)$,

$$
\begin{aligned}
J_{4} \leq & C \cdot w^{q}(B)^{((1 / q)-(\kappa / p))} \sum_{k=1}^{\infty}\left(1+\frac{r}{\rho\left(x_{0}\right)}\right)^{N \cdot\left(N_{0} /\left(N_{0}+1\right)\right)}\left(1+\frac{2^{k+1} r}{\rho\left(x_{0}\right)}\right)^{-N} \\
& \times \frac{1}{\left|2^{k+1} B\right|^{1-(\alpha / d)}} \int_{2^{k+1} B}\left|b(y)-b_{B}\right||f(y)| \mathrm{d} y .
\end{aligned}
$$

Let us consider each term in the sum of (111) separately. For each integer $k \geq 1$,

$$
\begin{aligned}
& \frac{1}{\left|2^{k+1} B\right|^{1-(\alpha / d)}} \int_{2^{k+1} B}\left|b(y)-b_{B}\right||f(y)| \mathrm{d} y \\
& \leq \frac{1}{\left|2^{k+1} B\right|^{1-(\alpha / d)}} \int_{2^{k+1} B}\left|b(y)-b_{2^{k+1} B}\right||f(y)| \mathrm{d} y \\
& +\frac{1}{\left|2^{k+1} B\right|^{1-(\alpha / d)}} \int_{2^{k+1} B}\left|b_{2^{k+1} B}-b_{B}\right||f(y)| \mathrm{d} y .
\end{aligned}
$$

By using Hölder's inequality, the first term of the expression (112) is bounded by

$$
\begin{gathered}
\frac{1}{\left|2^{k+1} B\right|^{1-(\alpha / d)}}\left(\int_{2^{k+1} B}|f(y)|^{p} w^{p}(y) \mathrm{d} y\right)^{1 / p} \\
\cdot\left(\int_{2^{k+1} B}\left|b(y)-b_{2^{k+1} B}\right|^{p^{\prime}} w(y)^{-p^{\prime}} \mathrm{d} y\right)^{1 / p^{\prime}} \\
\leq C\|f\|_{L_{\rho, \theta}^{p, k}\left(w^{p}, w^{q}\right)} \cdot \frac{w^{q}\left(2^{k+1} B\right)^{\kappa / p}}{\left|2^{k+1} B\right|^{1-(\alpha / d)}}\left(1+\frac{2^{k+1} r}{\rho\left(x_{0}\right)}\right)^{\theta} \\
\cdot\left(\int_{2^{k+1} B}\left|b(y)-b_{2^{k+1} B}\right|^{p^{\prime}} w(y)^{-p^{\prime}} \mathrm{d} y\right)^{1 / p^{\prime}} .
\end{gathered}
$$

Since $w \in A_{p, q}^{\rho, \theta^{\prime}}$ with $0<\theta^{\prime}<\infty$ and $1<p<q<\infty$, then by Lemma 5 , we know that $w^{-p^{\prime}} \in A_{t^{\prime}}^{\rho, \theta^{\prime} \cdot\left(1 /\left((1 / q)+\left(1 / p^{\prime}\right)\right)\right)}$ with $t^{\prime}=1+p^{\prime} / q$. If we denote $v=w^{-p^{\prime}}$, then $v \in A_{t^{\prime}}^{\rho, \theta^{\prime} \cdot\left(1 /\left((1 / q)+\left(1 / p^{\prime}\right)\right)\right)} \subset A_{p^{\prime}}^{\rho, \infty}$ because $t^{\prime}=1+\left(p^{\prime} / q\right)<p^{\prime}$. This fact together with Lemma 7 implies

$$
\begin{aligned}
& \left(\int_{2^{k+1} B}\left|b(y)-b_{2^{k+1} B}\right|^{p^{\prime}} v(y) \mathrm{d} y\right)^{1 / p^{\prime}} \\
& \quad \leq C_{b} \cdot v\left(2^{k+1} B\right)^{1 / p^{\prime}}\left(1+\frac{2^{k+1} r}{\rho\left(x_{0}\right)}\right)^{\mu} \\
& \quad=C_{b} \cdot\left(\int_{2^{k+1} B} w(y)^{-p^{\prime}} \mathrm{d} y\right)^{1 / p^{\prime}}\left(1+\frac{2^{k+1} r}{\rho\left(x_{0}\right)}\right)^{\mu} \\
& \leq C_{b, w} \cdot \frac{\left|2^{k+1} B\right|^{1-(\alpha / d)}}{w^{q}\left(2^{k+1} B\right)^{1 / q}}\left(1+\frac{2^{k+1} r}{\rho\left(x_{0}\right)}\right)^{\theta^{\prime}}\left(1+\frac{2^{k+1} r}{\rho\left(x_{0}\right)}\right)^{\mu} .
\end{aligned}
$$

Therefore, the first term of the expression (112) can be bounded by a constant times

$$
\frac{w^{q}\left(2^{k+1} B\right)^{\kappa / p}}{w^{q}\left(2^{k+1} B\right)^{1 / q}}\left(1+\frac{2^{k+1} r}{\rho\left(x_{0}\right)}\right)^{\theta+\theta^{\prime}+\mu} .
$$

Since $b \in \mathrm{BMO}_{\rho, \theta^{\prime \prime}}\left(\mathbb{R}^{d}\right)$ with $0<\theta^{\prime \prime}<\infty$, then by Lemma 8 , Hölder's inequality, and the $A_{p, q}^{\rho, \theta^{\prime}}$ condition on $w$, the latter term of the expression (112) can be estimated by

$$
\begin{aligned}
& C_{b}(k+1)\left(1+\frac{2^{k+1} r}{\rho\left(x_{0}\right)}\right)^{\theta^{\prime \prime}} \frac{1}{\left|2^{k+1} B\right|^{1-(\alpha / d)}} \int_{2^{k+1} B}|f(y)| \mathrm{d} y \\
& \leq C_{b}(k+1)\left(1+\frac{2^{k+1} r}{\rho\left(x_{0}\right)}\right)^{\theta^{\prime \prime}} \frac{1}{\left|2^{k+1} B\right|^{1-(\alpha / d)}} \\
& \cdot\left(\int_{2^{k+1} B}|f(y)|^{p} w^{p}(y) \mathrm{d} y\right)^{1 / p}\left(\int_{2^{k+1} B} w(y)^{-p^{\prime}} \mathrm{d} y\right)^{1 / p^{\prime}} \\
& \leq C\|f\|_{L_{\rho, \theta}^{p, k}\left(w^{p}, w^{q}\right)} \cdot(k+1) \frac{w^{q}\left(2^{k+1} B\right)^{\kappa / p}}{w^{q}\left(2^{k+1} B\right)^{1 / q}}\left(1+\frac{2^{k+1} r}{\rho\left(x_{0}\right)}\right)^{\theta+\theta^{\prime}+\theta^{\prime \prime}} .
\end{aligned}
$$


Consequently,

$$
\begin{aligned}
& \frac{1}{\left|2^{k+1} B\right|^{1-(\alpha / d)}} \int_{2^{k+1} B}\left|b(y)-b_{B}\right||f(y)| \mathrm{d} y \\
& \leq C\|f\|_{L_{\rho, \theta}^{p, \kappa}\left(w^{p}, w^{q}\right)} \cdot(k+1) \frac{w^{q}\left(2^{k+1} B\right)^{\kappa / p}}{w^{q}\left(2^{k+1} B\right)^{1 / q}}\left(1+\frac{2^{k+1} r}{\rho\left(x_{0}\right)}\right)^{\theta+\theta^{\prime}+\theta^{\prime \prime}+\mu} .
\end{aligned}
$$

Substituting the above inequality (117) into (111), we thus obtain

$$
\begin{aligned}
J_{4} \leq & C\|f\|_{L_{\rho, \theta}^{p, k}\left(w^{p}, w^{q}\right)} \cdot w^{q}(B)^{(1 / q)-(\kappa / p)} \\
& \cdot \sum_{k=1}^{\infty}(k+1)\left(1+\frac{r}{\rho\left(x_{0}\right)}\right)^{N \cdot\left(N_{0} /\left(N_{0}+1\right)\right)}\left(1+\frac{2^{k+1} r}{\rho\left(x_{0}\right)}\right)^{-N} \\
& \times \frac{1}{w^{q}\left(2^{k+1} B\right)^{((1 / q)-(\kappa / p))}}\left(1+\frac{2^{k+1} r}{\rho\left(x_{0}\right)}\right)^{\theta+\theta^{\prime}+\theta^{\prime \prime}+\mu} \\
= & C\|f\|_{L_{\rho, \theta}^{p, \kappa}\left(w^{p}, w^{q}\right)}\left(1+\frac{r}{\rho\left(x_{0}\right)}\right)^{N \cdot\left(N_{0} /\left(N_{0}+1\right)\right)} \\
& \times \sum_{k=1}^{\infty}(k+1) \frac{w^{q}(B)^{((1 / q)-(\kappa / p))}}{w^{q}\left(2^{k+1} B\right)^{((1 / q)-(\kappa / p))}}\left(1+\frac{2^{k+1} r}{\rho\left(x_{0}\right)}\right)^{-N+\theta+\theta^{\prime}+\theta^{\prime \prime}+\mu} \cdot \theta^{\prime \prime} .
\end{aligned}
$$

Note that $w^{q} \in A_{t}^{\rho, \theta^{\prime} \cdot\left(1 /\left((1 / q)+\left(1 / p^{\prime}\right)\right)\right)}$ with $t=1+q / p^{\prime}$. A further application of (25) yields

$$
\begin{aligned}
J_{4} \leq & C\|f\|_{L_{\rho, \theta}^{p, \kappa}\left(w^{p}, w^{q}\right)}\left(1+\frac{r}{\rho\left(x_{0}\right)}\right)^{N \cdot\left(N_{0} /\left(N_{0}+1\right)\right)} \\
& \cdot \sum_{k=1}^{\infty}(k+1)\left(\frac{|B|}{\left|2^{k+1} B\right|}\right)^{\delta((1 / q)-(\kappa / p))} \\
& \times\left(1+\frac{2^{k+1} r}{\rho\left(x_{0}\right)}\right)^{\eta(1 / q)-(\kappa / p)}\left(1+\frac{2^{k+1} r}{\rho\left(x_{0}\right)}\right)^{-N+\theta+\theta^{\prime}+\theta^{\prime \prime}+\mu} \\
= & C\|f\|_{L_{\rho, \theta}^{p, k}\left(w^{p}, w^{q}\right)}\left(1+\frac{r}{\rho\left(x_{0}\right)}\right)^{N \cdot\left(N_{0} /\left(N_{0}+1\right)\right)} \\
& \cdot \sum_{k=1}^{\infty}(k+1)\left(\frac{|B|}{\left|2^{k+1} B\right|}\right)^{\delta((1 / q)-(\kappa / p))} \\
& \times\left(1+\frac{2^{k+1} r}{\rho\left(x_{0}\right)}\right)^{-N+\theta+\theta^{\prime}+\theta^{\prime \prime}+\mu+\eta((1 / q)-(\kappa / p))} \cdot \theta^{\prime \prime} .
\end{aligned}
$$

Hence, combining the above estimates for $J_{3}$ and $J_{4}$, we conclude that

$$
\begin{aligned}
J_{2} \leq J_{3} & +J_{4} \leq C\|f\|_{L_{\rho, \theta}^{p, \kappa}\left(w^{p}, w^{q}\right)}\left(1+\frac{r}{\rho\left(x_{0}\right)}\right)^{\mu+N \cdot\left(N_{0} /\left(N_{0}+1\right)\right)} \\
& \times \sum_{k=1}^{\infty}(k+1)\left(\frac{|B|}{\left|2^{k+1} B\right|}\right)^{\delta((1 / q)-(\kappa / p))} \\
& \cdot\left(1+\frac{2^{k+1} r}{\rho\left(x_{0}\right)}\right)^{-N+\theta+\theta^{\prime}+\theta^{\prime \prime}+\mu+\eta((1 / q)-(\kappa / p))} \cdot \theta^{\prime \prime} .
\end{aligned}
$$

By choosing $N$ large enough so that $N>\theta+\theta^{\prime}+\theta^{\prime \prime}+\mu+\eta((1 / q)-(\kappa / p))$, we thus have

$$
\begin{aligned}
J_{2} \leq & C\left(1+\frac{r}{\rho\left(x_{0}\right)}\right)^{\mu+N \cdot\left(N_{0} /\left(N_{0}+1\right)\right)} \\
& \cdot \sum_{k=1}^{\infty}(k+1)\left(\frac{|B|}{\left|2^{k+1} B\right|}\right)^{\delta((1 / q)-(\kappa / p))} \\
\leq & C\left(1+\frac{r}{\rho\left(x_{0}\right)}\right)^{\mu+N \cdot\left(N_{0} /\left(N_{0}+1\right)\right)},
\end{aligned}
$$

where the last series is convergent since $0<\kappa<(p / q)$. Finally, collecting the above estimates for $J_{1}$ and $J_{2}$, and letting $\vartheta=\max \left\{\vartheta^{\prime}, \mu+N \cdot\left(N_{0} /\left(N_{0}+1\right)\right)\right\}$, we obtain the desired inequality (99). The proof of Theorem 6 is finished.

The higher-order commutators generated by $\mathrm{BMO}_{\rho, \infty}\left(\mathbb{R}^{d}\right)$ functions $b$ and the fractional integrals $\mathscr{I}_{\alpha}$ are usually defined by

$$
\left\{\begin{array}{l}
{\left[b, \mathscr{I}_{\alpha}\right]_{m} f(x):=\int_{\mathbb{R}^{n}}[b(x)-b(y)]^{m} \mathscr{K}_{\alpha}(x, y) f(y) \mathrm{d} y, \quad x \in \mathbb{R}^{d} ;} \\
0<\alpha<d, \quad m=1,2,3, \ldots
\end{array}\right.
$$

Obviously, $\left[b, \mathscr{I}_{\alpha}\right]_{1}=\left[b, \mathscr{I}_{\alpha}\right]$ which is just the linear commutator (40) and

$$
\left[b, \mathscr{I}_{\alpha}\right]_{m}=\left[b,\left[b, \mathscr{I}_{\alpha}\right]_{m-1}\right], \quad m=2,3, \ldots
$$

By induction on $m$, we are able to show that the conclusion of Theorem 6 also holds for the higher-order commutators $\left[b, \mathscr{I}_{\alpha}\right]_{m}$ with $m \geq 2$. The details are omitted here.

Theorem 8. Let $0<\alpha<d, 1<p<d / \alpha, 1 / q=1 / p-\alpha / d$, and $w \in A_{p, q}^{\rho, \infty}$. If $V \in R H_{s}$ with $s \geq d / 2$ and $0<\kappa<p / q$, then for any positive integer $m \geq 2$, the higher order commutators $\left[b, \mathscr{I}_{\alpha}\right]_{m}$ are bounded from $L_{\rho, \infty}^{p, \kappa}\left(w^{p}, w^{q}\right)$ into $L^{q,(\kappa q) / p}\left(w^{q}\right)$, whenever $b \in B M O_{\rho, \infty}\left(\mathbb{R}^{d}\right)$.

\section{Proof of Theorem 7}

The following lemma plays a key role in the proof of our main theorem, which can be found in Proposition 8 of [20] (see also [7], Lemma 3.7). 
Lemma 9 (see [20]). Let $V \in R H_{s}$ with $s \geq d / 2$ and $0<\alpha<d$. For every positive integer $N \geq 1$, there exists a positive constant $C_{N}>0$ such that, for all $x$ and $y$ in $\mathbb{R}^{d}$ and for some fixed $0<\varepsilon \leq 1$,

$$
\left|\mathscr{K}_{\alpha}(x, z)-\mathscr{K}_{\alpha}(y, z)\right| \leq C_{N}\left(1+\frac{|x-z|}{\rho(x)}\right)^{-N} \frac{|x-y|^{\varepsilon}}{|x-z|^{d-\alpha+\varepsilon}}
$$

whenever $|x-y| \leq|x-z| / 2$.

Proof of Theorem 7. For an arbitrary ball $B=B\left(x_{0}, r\right)$ in $\mathbb{R}^{d}$ and $0<\alpha<d$, it suffices to prove that the following inequality

$$
\frac{1}{|B|} \int_{B}\left|\mathscr{I}_{\alpha} f(x)-\left(\mathscr{I}_{\alpha} f\right)_{B}\right| \mathrm{d} x \leq C \cdot\left(1+\frac{r}{\rho\left(x_{0}\right)}\right)^{\vartheta},
$$

holds for any $f \in L_{\rho, \infty}^{p, \kappa}\left(w^{p}, w^{q}\right)$ with $1<p<q<\infty$ and $\kappa=p / q$, where $\left(\mathscr{I}_{\alpha} f\right)_{B}$ denotes the average of $\mathscr{I}_{\alpha} f$ over $B_{\text {; }}$ Suppose that $f \in L_{\rho, \theta}^{p, \kappa}\left(w^{p}, w^{q}\right)$ for some $\theta>0$ and $w \in A_{p, q}^{\rho, \theta^{\prime}}$ for some $\theta^{\prime}>0$. Decompose the function $f$ as $f=f_{1}+f_{2}$, where $f_{1}=f \cdot \chi_{4 B}, f_{2}=f \cdot \chi_{(4 B)}$, and $4 B=B\left(x_{0}, 4 r\right)$. By the linearity of the $\mathscr{L}$-fractional integral operator $\mathscr{I}_{\alpha}$, the left-hand side of (125) can be divided into two parts. That is,

$$
\begin{aligned}
\frac{1}{|B|} \int_{B}\left|\mathscr{I}_{\alpha} f(x)-\left(\mathscr{I}_{\alpha} f\right)_{B}\right| \mathrm{d} x \leq & \frac{1}{|B|} \int_{B}\left|\mathscr{I}_{\alpha} f_{1}(x)-\left(\mathscr{I}_{\alpha} f_{1}\right)_{B}\right| \mathrm{d} x \\
& +\frac{1}{|B|} \int_{B}\left|\mathscr{I}_{\alpha} f_{2}(x)-\left(\mathscr{I}_{\alpha} f_{2}\right)_{B}\right| \mathrm{d} x \\
:= & K_{1}+K_{2} .
\end{aligned}
$$

First, let us consider the term $K_{1}$. Applying the weighted $\left(L^{p}, L^{q}\right)$-boundedness of $\mathscr{I}_{\alpha}$ (see Theorem 1$)$ and Hölder's inequality, we obtain

$$
\begin{aligned}
K_{1} & \leq \frac{2}{|B|} \int_{B}\left|\mathscr{I}_{\alpha} f_{1}(x)\right| \mathrm{d} x \\
& \leq \frac{2}{|B|}\left(\int_{B}\left|\mathscr{I}_{\alpha} f_{1}(x)\right|^{q} w^{q}(x) \mathrm{d} x\right)^{1 / q}\left(\int_{B} w(x)^{-q^{\prime}} d x\right)^{1 / q^{\prime}} \\
& \leq \frac{C}{|B|}\left(\int_{4 B}|f(x)|^{p} w^{p}(x) \mathrm{d} x\right)^{1 / p}\left(\int_{B} w(x)^{-q^{\prime}} \mathrm{d} x\right)^{1 / q^{\prime}} \\
& \leq C\|f\|_{L_{\rho, \theta}^{p, \kappa}\left(w^{p}, w^{q}\right)} \cdot \frac{w^{q}(4 B)^{\kappa / p}}{|B|}\left(\int_{B} w(x)^{-q^{\prime}} \mathrm{d} x\right)^{1 / q^{\prime}}\left(1+\frac{4 r}{\rho\left(x_{0}\right)}\right)^{\theta} .
\end{aligned}
$$

Since $q^{\prime}<p^{\prime}$, by Hölder's inequality, it is easy to check that

$$
\left(\frac{1}{|B|} \int_{B} w(x)^{-q^{\prime}} \mathrm{d} x\right)^{1 / q^{\prime}} \leq\left(\frac{1}{|B|} \int_{B} w(x)^{-p^{\prime}} \mathrm{d} x\right)^{1 / p^{\prime}} .
$$

Moreover, since $w$ is a weight in the class $A_{p, q}^{\rho, \theta^{\prime}}$, one has

$$
\begin{aligned}
& \left(\frac{1}{|B|} \int_{B} w(x)^{q} \mathrm{~d} x\right)^{1 / q}\left(\frac{1}{|B|} \int_{B} w(x)^{-q^{\prime}} \mathrm{d} x\right)^{1 / q^{\prime}} \\
& \quad \leq\left(\frac{1}{|B|} \int_{B} w(x)^{q} \mathrm{~d} x\right)^{1 / q}\left(\frac{1}{|B|} \int_{B} w(x)^{-p^{\prime}} \mathrm{d} x\right)^{1 / p^{\prime}} \\
& \quad \leq C \cdot\left(1+\frac{r}{\rho\left(x_{0}\right)}\right)^{\theta^{\prime}}
\end{aligned}
$$

which implies

$$
\left(\int_{B} w(x)^{-q^{\prime}} \mathrm{d} x\right)^{1 / q^{\prime}} \leq C \cdot\left(1+\frac{r}{\rho\left(x_{0}\right)}\right)^{\theta^{\prime}} \frac{|B|}{w^{q}(B)^{1 / q}} .
$$

Also observe that $w^{q} \in A_{t}^{\rho, \theta^{\prime} \cdot\left(1 /\left((1 / q)+\left(1 / p^{\prime}\right)\right)\right)}$ with $t=1+q / p^{\prime}$. Using the inequalities (66) and (130) and noting the fact that $\kappa=p / q$, we have

$$
\begin{aligned}
K_{1} & \leq C\|f\|_{L_{\rho, \theta}^{p, \kappa}\left(w^{p}, w^{q}\right)} \cdot \frac{w^{q}(4 B)^{1 / q}}{w^{q}(B)^{1 / q}}\left(1+\frac{4 r}{\rho\left(x_{0}\right)}\right)^{\theta}\left(1+\frac{r}{\rho\left(x_{0}\right)}\right)^{\theta^{\prime}} \\
& \leq C\|f\|_{L_{\rho, \theta}^{p, \kappa}\left(w^{p}, w^{q}\right)}\left(1+\frac{4 r}{\rho\left(x_{0}\right)}\right)^{2 \theta^{\prime}}\left(1+\frac{4 r}{\rho\left(x_{0}\right)}\right)^{\theta}\left(1+\frac{r}{\rho\left(x_{0}\right)}\right)^{\theta^{\prime}} \\
& \leq C \cdot\left(1+\frac{r}{\rho\left(x_{0}\right)}\right)^{\vartheta^{\prime}},
\end{aligned}
$$

where $\vartheta^{\prime}:=3 \theta^{\prime}+\theta$. Now we turn to estimate $K_{2}$. For any $x \in B\left(x_{0}, r\right)$,

$$
\begin{aligned}
& \left|\mathscr{I}_{\alpha} f_{2}(x)-\left(\mathscr{I}_{\alpha} f_{2}\right)_{B}\right|=\left|\frac{1}{|B|} \int_{B}\left[\mathscr{I}_{\alpha} f_{2}(x)-\mathscr{I}_{\alpha} f_{2}(y)\right] \mathrm{d} y\right| \\
& =\left|\frac{1}{|B|} \int_{B}\left\{\int_{(4 B)^{c}}\left[\mathscr{K}_{\alpha}(x, z)-\mathscr{K}_{\alpha}(y, z)\right] f(z) \mathrm{d} z\right\} \mathrm{d} y\right| \\
& \leq \frac{1}{|B|} \int_{B}\left\{\int_{(4 B)^{c}}\left|\mathscr{K}_{\alpha}(x, z)-\mathscr{K}_{\alpha}(y, z)\right| \cdot|f(z)| \mathrm{d} z\right\} \mathrm{d} y .
\end{aligned}
$$

Next note that, by a purely geometric argument, one has

$$
\begin{aligned}
& |x-y| \leq \frac{|x-z|}{2}, \\
& |x-z| \approx\left|x_{0}-z\right|,
\end{aligned}
$$

whenever $x, y \in B$ and $z \in(4 B)^{c}$. This fact along with Lemma 9 yields 


$$
\begin{aligned}
\left|\mathscr{I}_{\alpha} f_{2}(x)-\left(\mathscr{I}_{\alpha} f_{2}\right)_{B}\right| & \leq \frac{C_{N}}{|B|} \int_{B}\left\{\int_{(4 B)^{c}}\left(1+\frac{|x-z|}{\rho(x)}\right)^{-N} \frac{|x-y|^{\varepsilon}}{|x-z|^{d-\alpha+\varepsilon}} \cdot|f(z)| \mathrm{d} z\right\} \mathrm{d} y \\
& \leq C_{N, d} \int_{(4 B)^{c}}\left(1+\frac{\left|x_{0}-z\right|}{\rho(x)}\right)^{-N} \frac{r^{\varepsilon}}{\left|x_{0}-z\right|^{d-\alpha+\varepsilon}} \cdot|f(z)| \mathrm{d} z \\
& =C_{N, d} \sum_{k=2}^{\infty} \int_{2^{k+1} B \mid 2^{k} B}\left(1+\frac{\left|x_{0}-z\right|}{\rho(x)}\right)^{-N} \frac{r^{\varepsilon}}{\left|x_{0}-z\right|^{d-\alpha+\varepsilon}} \cdot|f(z)| \mathrm{d} z \\
& \leq C_{N, d} \sum_{k=2}^{\infty} \frac{1}{2^{k \varepsilon}} \cdot \frac{1}{\left|2^{k+1} B\right|^{1-(\alpha / d)}} \int_{2^{k+1} B \backslash 2^{k} B}\left(1+\frac{2^{k} r}{\rho(x)}\right)^{-N}|f(z)| \mathrm{d} z .
\end{aligned}
$$

Furthermore, by using Hölder's inequality, (4) and $A_{p, q}^{\rho, \theta^{\prime}}$ condition on $w$, we get that, for any $x \in B\left(x_{0}, r\right)$,

$$
\begin{aligned}
\left|\mathscr{I}_{\alpha} f_{2}(x)-\left(\mathscr{I}_{\alpha} f_{2}\right)_{B}\right| \leq & C \sum_{k=2}^{\infty} \frac{1}{2^{k \varepsilon}} \cdot \frac{1}{\left|2^{k+1} B\right|^{1-(\alpha / d)}}\left(1+\frac{r}{\rho\left(x_{0}\right)}\right)^{N \cdot\left(N_{0} /\left(N_{0}+1\right)\right)}\left(1+\frac{2^{k+1} r}{\rho\left(x_{0}\right)}\right)^{-N} \\
& \times\left(\int_{2^{k+1} B}|f(z)|^{p} w^{p}(z) \mathrm{d} z\right)^{1 / p}\left(\int_{2^{k+1} B} w(z)^{-p^{\prime}} \mathrm{d} z\right)^{1 / p^{\prime}} \\
\leq & C\|f\|_{L_{\rho, \theta}^{p, k}\left(w^{p}, w^{q}\right)} \sum_{k=2}^{\infty} \frac{1}{2^{k \varepsilon}} \cdot\left(1+\frac{r}{\rho\left(x_{0}\right)}\right)^{N \cdot\left(N_{0} /\left(N_{0}+1\right)\right)}\left(1+\frac{2^{k+1} r}{\rho\left(x_{0}\right)}\right)^{-N} \\
& \times \frac{w^{q}\left(2^{k+1} B\right)^{k / p}}{w^{q}\left(2^{k+1} B\right)^{1 / q}}\left(1+\frac{2^{k+1} r}{\rho\left(x_{0}\right)}\right)^{\theta}\left(1+\frac{2^{k+1} r}{\rho\left(x_{0}\right)}\right)^{\theta^{\prime}} \\
= & C\|f\|_{L_{\rho, \theta}^{p, k}\left(w^{p}, w^{q}\right)} \sum_{k=2}^{\infty} \frac{1}{2^{k \varepsilon}} \cdot\left(1+\frac{r}{\rho\left(x_{0}\right)}\right)^{N \cdot\left(N_{0} /\left(N_{0}+1\right)\right)}\left(1+\frac{2^{k+1} r}{\rho\left(x_{0}\right)}\right)^{-N+\theta+\theta^{\prime}},
\end{aligned}
$$

where the last equality is due to the assumption $\kappa=p / q$.

From the pointwise estimate (135), it readily follows that

$$
\begin{aligned}
K_{2}= & \frac{1}{|B|} \int_{B}\left|\mathscr{I}_{\alpha} f_{2}(x)-\left(\mathscr{I}_{\alpha} f_{2}\right)_{B}\right| \mathrm{d} x \\
\leq & C\|f\|_{L_{\rho, \theta}^{p, k}\left(w^{p}, w^{q}\right)} \sum_{k=2}^{\infty} \frac{1}{2^{k \varepsilon}} \cdot\left(1+\frac{r}{\rho\left(x_{0}\right)}\right)^{N \cdot\left(N_{0} /\left(N_{0}+1\right)\right)} \\
& \cdot\left(1+\frac{2^{k+1} r}{\rho\left(x_{0}\right)}\right)^{-N+\theta+\theta^{\prime}} .
\end{aligned}
$$

Now $N$ can be chosen sufficiently large so that $N>\theta+\theta^{\prime}$, and hence, the above series is convergent. Therefore,

$$
\begin{aligned}
K_{2} \leq & C\left(1+\frac{r}{\rho\left(x_{0}\right)}\right)^{N \cdot\left(N_{0} /\left(N_{0}+1\right)\right)} \sum_{k=2}^{\infty} \frac{1}{2^{k \varepsilon}} \\
& \leq C\left(1+\frac{r}{\rho\left(x_{0}\right)}\right)^{N \cdot\left(N_{0} /\left(N_{0}+1\right)\right)} .
\end{aligned}
$$

Fix this $N$ and set $\vartheta=\max \left\{\vartheta^{\prime}, N \cdot\left(N_{0} / N_{0}+1\right)\right\}$. Finally, combining the above estimates for $K_{1}$ and $K_{2}$, the inequality (125) is proved, and then the proof of Theorem 7 is finished.

\section{Data Availability}

No data were used to support this study. 


\section{Conflicts of Interest}

The author declares that there are no conflicts of interest regarding the publication of this paper.

\section{Acknowledgments}

This work was done while the author was visiting Memorial University of Newfoundland in Canada. He wishes to thank Prof. Jie Xiao for the invitation and the warm hospitality during his visit.

\section{References}

[1] Z. Shen, " $L^{p}$ estimates for Schrödinger operators with certain potentials," Annales de l'institut Fourier, vol. 45, no. 2, pp. 513-546, 1995.

[2] B. Bongioanni, E. Harboure, and O. Salinas, "Classes of weights related to Schrödinger operators," Journal of Mathematical Analysis and Applications, vol. 373, no. 2, pp. 563579, 2011.

[3] J. Dziubański, G. Garrigós, T. Martínez, J. L. Torrea, and J. Zienkiewicz, "BMO spaces related to Schrödinger operators with potentials satisfying a reverse Hölder inequality," Mathematische Zeitschrift, vol. 249, no. 2, pp. 329-356, 2005.

[4] B. Bongioanni, E. Harboure, and O. Salinas, "Weighted inequalities for commutators of Schrödinger-Riesz transforms," Journal of Mathematical Analysis and Applications, vol. 392, no. 1, pp. 6-22, 2012.

[5] B. Bongioanni, E. Harboure, and O. Salinas, "Commutators of riesz transforms related to schrödinger operators," Journal of Fourier Analysis and Applications, vol. 17, no. 1, pp. 115-134, 2011.

[6] L. Grafakos, Classical Fourier Analysis, Springer-Verlag, Berlin, Germany, 3rd edition, 2014.

[7] L. Tang, "Weighted norm inequalities for Schrödinger type operators," Forum Mathematicum, vol. 27, no. 4, pp. 2491-2532, 2015.

[8] B. Muckenhoupt and R. Wheeden, "Weighted norm inequalities for fractional integrals," Transactions of the American Mathematical Society, vol. 192, p. 261, 1974.

[9] R. Johnson and C. J. Neugebauer, "Change of variable results for $A_{p}$-and reverse holder $\mathrm{RH}_{r}$-classes," Transactions of the American Mathematical Society, vol. 328, no. 2, pp. 639-666, 1991.

[10] F. John and L. Nirenberg, "On functions of bounded mean oscillation," Communications on Pure and Applied Mathematics, vol. 14, no. 3, pp. 415-426, 1961.

[11] T. A. Bui, "Weighted estimates for commutators of some singular integrals related to Schrödinger operators," Bulletin des Sciences Mathématiques, vol. 138, no. 2, pp. 270-292, 2014.

[12] C. Segovia and J. L. Torrea, "Weighted inequalities for commutators of fractional and singular integrals," Publicacions Matemàtiques, vol. 35, pp. 209-235, 1991.

[13] G. X. Pan and L. Tang, "Boundedness for some Schrödinger type operators on weighted Morrey spaces," Journal of Function Spaces, vol. 2014, Article ID 878629, 10 pages, 2014.

[14] L. Tang and J. Dong, "Boundedness for some Schrödinger type operators on Morrey spaces related to certain nonnegative potentials," Journal of Mathematical Analysis and Applications, vol. 355, no. 1, pp. 101-109, 2009.

[15] Y. Komori and S. Shirai, "Weighted Morrey spaces and a singular integral operator," Mathematische Nachrichten, vol. 282, no. 2, pp. 219-231, 2009.
[16] H. Wang, "Intrinsic square functions on the weighted Morrey spaces," Journal of Mathematical Analysis and Applications, vol. 396, no. 1, pp. 302-314, 2012.

[17] H. Wang, "Weak type estimates for intrinsic square functions on weighted Morrey spaces," Analysis in Theory and Applications, vol. 29, no. 2, pp. 104-119, 2013.

[18] H. Wang, "Weighted inequalities for fractional integral operators and linear commutators in the Morrey-type spaces," Journal of Inequalities and Applications, vol. 2017, no. 1, p. 33, 2017.

[19] B. Bongioanni, A. Cabral, and E. Harboure, "Extrapolation for classes of weights related to a family of operators and applications," Potential Analysis, vol. 38, no. 4, pp. 1207-1232, 2013.

[20] B. Bongioanni, A. Cabral, and E. Harboure, "Lerner's inequality associated to a critical radius function and applications," Journal of Mathematical Analysis and Applications, vol. 407, no. 1, pp. 35-55, 2013. 